\title{
EDDY CURRENT TEST PATTERN RECOGNITION PROGRAM
}

\author{
NOVEMBER 1968
}

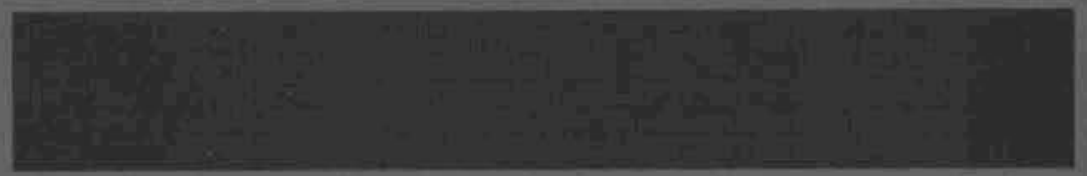

\section{AEC RESEARCH \& DEVELOPMENT REPORT}




\title{
LEGAL NOTICE
}

This repott wos prepared as an account of Government sponsored work. Neither the United States. nor the Commission, nor any person octing on beholf of the Commission:

A. Mokes any wartanty or cepresentation, expressed or implied, with respect to the occuracy, completeness, or usefulness of the information coniained in this report of that the use of any infarmation, apparatus, method, or process disclosed in this teport may not infringe privately owned rights; or

B. Assumes ony liabllities with respect to the use of, or for damages resulting from the use of any information, apporatus. method, or process disclosed in this report.

As used in the above, "person acting on behalf of the Commission" includes any employee, or contractor of the Cammission, on employee of such contractor, to the extent that such employeo or contractor of the Commission, or employes of such coniracton prepares, disseminates, or provides acress to. any infarmation putsuant to his employment of contract with the Commistion, or his employment with such controctor:

\author{
PACIFIC NORTHWEST LABORATORY \\ BICHLAND, WASHINGTON \\ operated by
}

BATTELLE MEMORIAL INSIITUTE

for the

UNITED STATES ATOMIC ENERGY COMMISSION UNDER CONTRACT AT(45-1)-1830 


\title{
33679000612137
}

\author{
BNWL-942 \\ UC-37, Instruments \\ EDDY CURRENT TEST PATTERN RECOGNITION PROGRAM \\ by \\ R. N. Ord \\ Nondestructive Testing Department \\ and
}

R. L. Richardson

Applied Mathematics Department

Systems and Electronics Division

November 1968

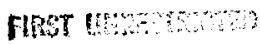

otwhing ins?

082788 
Printed in the United States of America Available from

Clearinghouse for Federal Scientific and Technical Information National Bureau of Standards, U. S. Department of Commerce Springfield, Virginia 22151

Price: Printed Copy $\$ 3.00$; Microfiche $\$ 0.65$ 
ii

EDDY CURRENT TEST PATTERN RECOGNITION PROGRAM

\section{ABSTRACT}

The size and shape of a test pattern generated by an "eddy current" probe passing a flaw defect in metal tubing presents an opportunity to classify flaw patterns using automated techniques. The first step in this direction was the development of a mathematical model making use of the first two statistical moments to correlate an unknown pattern with a standard set of known patterns. Twelve of the 20 patterns se1ected at random were identified with the standard set of 23 patterns. The accumulation of experience with this process will lead to an improved set of standard patterns and consequent improvement in random pattern identification. The ultimate goal of this program will be the development of a test box for field use which will be used for "eddy current" incipient failure testing. 
TABLE OF CONTENTS

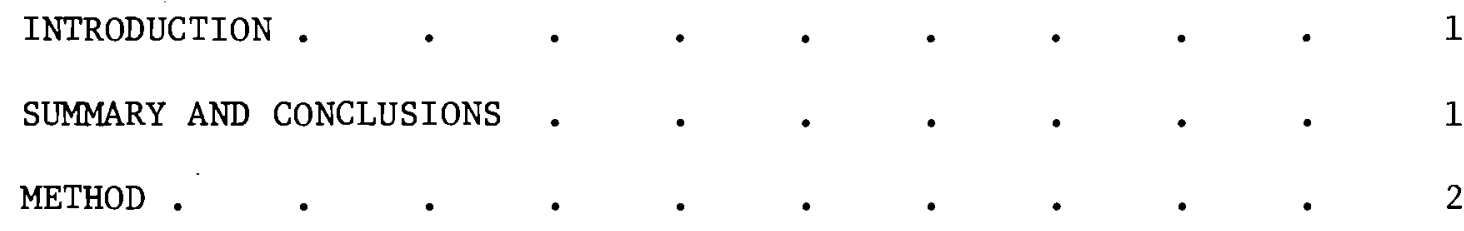

APPENDIX I

\section{APPENDIX I}

IDENTIFICATION OF CORRESPONDING STANDARD PATTERNS WHERE THE UNKNOWN PATTERNS CONTAIN READING NOISE

APPENDIX III

EDDY CURRENT TUBING FLAW PATTERNS, INTENTIONAL FLAWS • 35

APPENDIX IV

EDDY CURRENT TUBING FLAW PATTERNS, RANDOM FLAWS • • 38 APPENDIX V

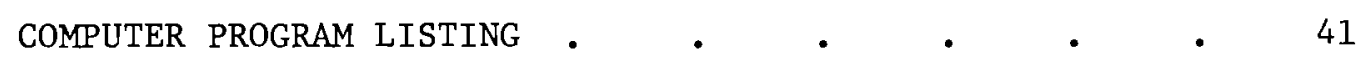

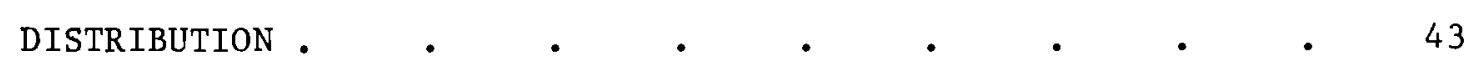




\title{
EDDY CURRENT TEST PATTERN RECOGNITION PROGRAM
}

\author{
R. N. Ord and R. L. Richardson
}

\section{INTRODUCTION}

The detection of flaw defects in metals using eddy current test equipment has become a routine procedure for such members as heat exchanger tubing, fuel sheath tubing, and many others. The most advanced single frequency eddy current test equipment systems present1y in use provide a readout composed of two variable dc voltages which form a complicated pattern (generally of figure eight shape) when presented on an X-Y oscilloscope. A continuous relationship exists between the shape and size of the patterns and the location and magnitude of the defects. The interpretation of test results is presently limited to the operator's ability to recognize this relationship supplemented by simple level sensing reject circuits. Quality assurance decisions, which are dependent upon the educated judgment and memory of different operators, suffer from a lack of uniformity. This is presently the limiting factor in improving the reproducibility of eddy current defect detection tests.

This report describes a method of test procedure simplification which wil1 substitute computerized pattern recognition for human pattern recognition. This has been brought about partially by the need for uniform, high quality standards that will be necessary to produce the metal products for the successful development, construction, and operation of new reactor concepts such as the liquid metal fast breeder reactor.

\section{SUMMARY AND CONCLUSIONS}

The ability to mathematically recognize and classify eddy current patterns has been demonstrated in this study. Of 20 patterns selected at random, twelve were identified with the set of 23 "standard patterns". 
The failure to identify eight of the 20 patterns selected at random would appear to be intolerable. However, the quality of these patterns was purposely chosen to make detection difficult. At the outset, no statistical error structure was available to help define the term "standard patterns". The procedure given in the body of the report is the initial phase of a program which will define standard patterns, perhaps eliminating those already chosen and adding new standard patterns which are found to be more frequently encountered. The process variables of linearity and gain offer no difficulty. However, some patterns must go unrecognized because of problens with "signal-to-noise ratio" and "zero adjustment" or "drift". Experience with the technique will indicate the kind of statistics which need to be incorporated in the development of a more sophisticated pattern recognition program.

\section{METHOD}

The technique derived in this report is to compare the size and shape correspondences between an unknown eddy current flaw pattern with a standard set of known patterns for the particular specimen geometry and eddy current test coil configuration. Two correlation coefficients are derived for each unknown with respect to one of the standard patterns. These correlation coefficients refer to the shape of the pattern correspondence $\left(C_{i j}\right)$ and the magnitude correspondence $\left(\mathrm{M}_{i j}\right)$. Also given is the product correspondence of these two correlation factors.

$$
P_{i j}=M_{i j} C_{i j}
$$

The result of the computer program is to derive a $P_{i j}, M_{i j}$ and $C_{i j}$ for each unknown pattern.

Analytical Development

Twenty-three patterns were provided as a basis for detection. The patterns were supplied on polar graph paper with the pole and polar axis being specified by the operator. Each pattern was represented mathematically by a set of measured values of pattern radius in inches and 
polar angle in degrees. The polar angle was taken every $10^{\circ}$ from the polar axis inclusive. At angles where the pattern was double valued, the largest radius was taken. Let the given pattern sets be defined

$$
\begin{array}{r}
\vec{R}_{i}=\left\{r_{1}\left(0^{\circ}\right), r_{2}\left(10^{\circ}\right), r_{3}\left(20^{\circ}\right), \ldots r_{36}\left(350^{\circ}\right)\right\}_{i}, \\
i=1,2,3, \ldots, 23 .
\end{array}
$$

where the $r_{k}$ are the product of kth measured radius and the sensitivity In volts per inch at which that pattern was recorded.

Nine of the patterns were selected at random and values of $\vec{R}_{i}$ measured again with intentional error in the measured values of radii. These pattern sets were similarly defined

$$
\begin{array}{r}
\vec{R}_{j}=\left\{r_{1}(0), r_{2}\left(10^{\circ}\right), r_{3}\left(20^{\circ}\right), \ldots, r_{36}\left(350^{\circ}\right)\right\} j \\
j=1,2,3, \ldots, 9 .
\end{array}
$$

Hereafter $r_{i j}$ is the $i$ th measured radius of the $j$ th pattern vector. Correspondence in shape between the patterns in (1) and the patterns in (2) were obtained by evaluating the correlation coefficients $\left(C_{i j}\right)$.

$$
C_{i j}=\left(\sum_{k=1}^{36} r_{i k} r_{j k}\right) /\left(\left|\vec{R}_{i}\right|\left|\vec{R}_{j}\right|\right), i=1,2, \ldots 23, j=1,2, \ldots, 9
$$

where

$$
\overrightarrow{\mathrm{R}}_{\ell}=\sqrt{\sum_{\mathrm{k}} \mathrm{r}_{\ell \mathrm{k}}^{2}}, \ell=i, j \text {. }
$$

It can be shown that $0 \leq C_{i j} \leq 1$ for all $i, j$.

Correspondences in magnitude between the patterns in (1) and the patterns in (2) were obtained by evaluating the magnitude coefficients $\left(M_{i j}\right)$.

$$
M_{i j}=1-|1-| \vec{R}_{j}|/| \vec{R}_{i}||, i-1,2, \ldots, 23, j=1,2, \ldots, 9 .
$$

It can be shown that $M_{i j} \leq 1$. 
Values of $\mathrm{C}_{i j}$ near unity indicate the patterns have the same shape. Values of $\mathrm{M}_{i j}$ near unity indicate the patterns have the same size. Let the product $P_{i j}$ be called the "correspondence" of pattern $i$ with pattern $j$ and be given by

$$
P_{i j}=M_{i j} C_{i j}
$$

Then values of $P_{i j}$ near unity indicate the pattern $i$ has the same size and shape as the pattern $j$, and are termed corresponding patterns. Computer program results are listed in Appendix I in the form of tables in which these correspondences are demonstrated. The unknown patterns $j=1,2,3, \ldots, 9$ were designated AA through II. Data pertaining to unknown patterns are listed under the "unknown" column. The next three columns list corresponding pattern data, the first column having highest correspondence, the second lesser correspondence, etc. The known patterns are numbered in the row "variable number." The kind of flaw producing the pattern is given in the row "variable name." The $\mathrm{C}_{i j}$ are given in the row "correlation." The $M_{i j}$ are given in the row " 1 - ABS(1-RX/RK)." The $P_{i j}$ are given in the row "product." The $\left|\vec{R}_{\ell}\right|$ are given in the row "magnitude." The next row of the table lists the sensitivity in volts/ inch at which each pattern was recorded. The following rows contain the measured values in inches of pattern radii. The first column lists the angle in degrees at which the pattern radii were measured.

Tests on pattern recognition were made more severe by obtaining new recordings of arbitrary patterns. Some patterns were repetitions of the original 23 patterns. All patterns were recorded on rectangular 
coordinate paper with no identification of the pole or polar axis except for the orientation. From a random selection of 20 patterns, 12 were identified as belonging to the 23 original patterns used as standards. of these 12 patterns, 11 were identified in the first column after the unknown column and one in the second column. These results are listed under Appendix II with the unknown patterns, labeled A through $\mathrm{T}$. You will note that some of the corresponding patterns were recorded at different sensitivities. This may account for reduced values of correlation coefficient since the different sized patterns were converted visually to digital data. The original 23 patterns are shown in Figures 1 through 23 of Appendix III. The 20 arbitrary patterns are shown in Figures 1 through 20 of Appendix IV. The computer program is given in Appendix $\mathrm{V}$.

These results indicate feasibility of the mathematical technique for identifying arbitrary patterns. This technique then becomes the basis of a proposal for the development of an automatic test system for use in the field which will bring about improved flaw identification using eddy current techniques. 
VARIAYLE NUMBER VARI IALLE NAME CORRELATION

1-ABS $(1-R X / R K)$

PKOOUCT

MAGN I TUDE

SENSITIVITY V/IN

DATA VECTOR - DEG
UNKINOWN

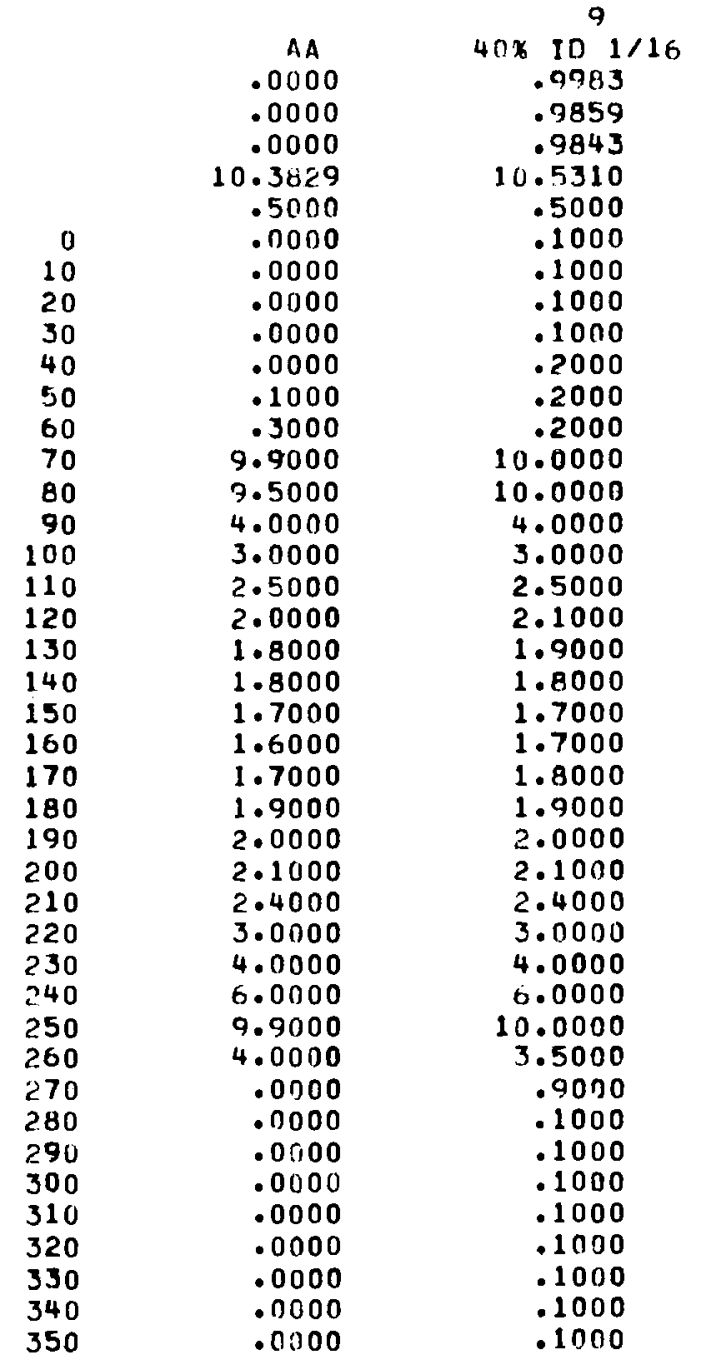

$M(2)$

${ }^{7} 3 / 10$

20\% I0 $3 / 10$

.7320

.6417
.4697

16.1793

.5000

2.0000

2.2000

3.1000

4.1000

6.0000

9.4000

19.4000

.2000

.2000

.2000

.3000

.3000

- 3000

.4000

.5000

.5000

.6000

.8000

.9000
1.1000

1.1000
1.8000

3.3000

5.4000

9.8000

18.5000

1.1000

1.1000

1.2000

1.2000

1.3000

1.3000

1.4000

1.5000

1.3000
$M(3)$

20\% $101 / 8$

.7065

.5433

.4273

.5000

.5000

- 000

.8000
.1000

1.2000

4. 5000

11.0060

22.0000

- 1000

1.5000

$1 \cdot 21000$

1.8000
1.8000

1.8000

1.9000

2.0000

$2 \cdot 1000$

c. 4000

c. $\$ 000$

3.2000

5.83000
5.5000

7.9000

12.3000

22. 3000

.0000

1.2000

.7000
.5000

.5000
.4000

.4000

.3000

.3000

.3000
$M(4)$

40* $10 \stackrel{2}{1 / 16}$

.7109

.4421

.3143
23.4840

1.0000

.0000

.0000

$.10 \cap 0$

1.2000
2.9000

2.9000
5.0000

33.5000

13.3000

.0000

.0000

.0000
.0000

.0000

.0000

.0000

.0000

.0000

.0000

- 1000

.1000
1.1000

2.2000

4.80170

8.2000

13.0000

1.3000

.6000

- 1000

.2000

- 1000

.0000

.0000

.0000

.0000

$M(5)$
4
$20 \% 003 / 16$
.3756
.6266
.2351
7.5600
.2000
.0060
.0000
.00110
.0090
.0000
.0000
.0000
.0000
.0000
10.0000
9.4000
9.0000
10.0000
10.0000
11.8000
.0000
.0000
.0000
.0000
.0000
.0000
.0000
.0000
.0000
.0000
9.9000
10.9000
7.2000
8.7000
10.0000
11.2050
12.9000
7.50100
.0000
.0000
.0000


VARIABLE NUMBER

VARIABLE NAME CORRELATION

1-ABS $(1-R X / R K)$

PRODUCT

MAGNI TUDE

SENSITIVITY V/IN

DATA VECTOR - DEG
UNKNOWN

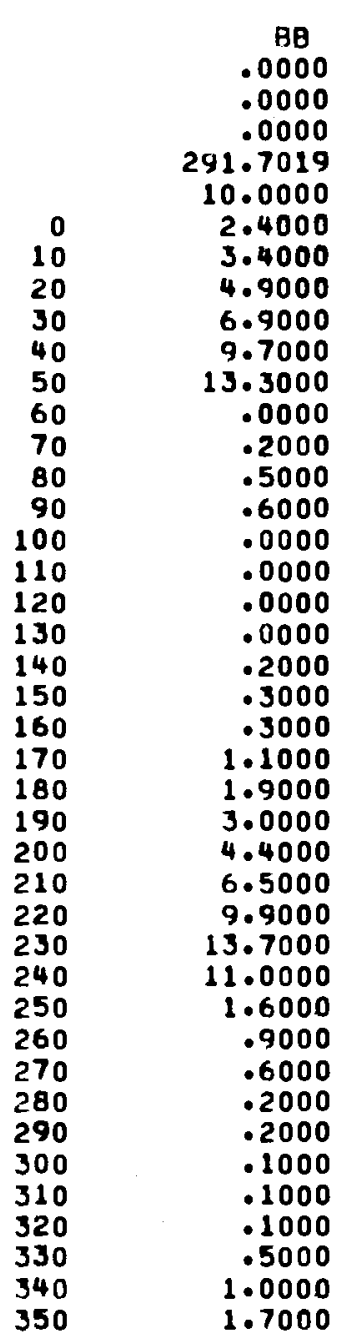

M(1)

$100 \% 1 / 8$

0.093

.9993
.9998

.9998

291.6316
10.0000

10.0000
2.4000

3.4000

4.9000

6.9000

9.8000
13.2000

13.2000
.0000

.0000

.0000

.0000

.0000

.0000

.0000

.0000

.0000
.0000

.0000

1.1000

1.9000

3.0000

4.5000

6.4000

9.9000

13.8000

11.0000

1.6000

.6000

.4000

.2000

.2000
.2000

.1000

- 1000

.1000

1.0000
$100 \times 3 / 16$

.9164

.5493
.5034

531.0273

10.0000
3.7000

5.2000

7.9000

11.8000

17.0000

24.1000

20.4000

1.9000

.8000
.6000

.6000

1000

.1000

.1000

.7000
.9000

.9000
1.1000

1.6000

2.2000

3.2000

4.9000

7.0000

10.4000

15.9000

24.5000

15.0000

.0000

.0000

-00u0

.0000

.0000

.0000

.0000
.0000

.0000
1.0000

1.3000

1.3000
2.2000
$M 13$

$80 \times 101 / 8$

.7968
-1.2848

-1.2848
-1.0238

88.8041
2.0000

2.0000
.0000

2.0000

4.0000

6.9000

11.0000

$18 \cdot 4000$
20.5000

.0000

.0000

.0000

.0000

.0000

.0000

.0000

.0000

.0000

.5000

1.0000
1.9000

4.0000

6.0000

10.1000

17.4000

17.4000
23.5000

.0000

.0000

.0000

.0000

.0000

.0000

.0000

.0000

.0000

.0000

.0000
.0000
$M(4)$

$40 \times 13$

.0733

$-14.0064$

$-1.0262$

18.2241

.2000

.8000

1.1000

1.3000

2.2000

4.2000
7.9000

7.9000
10.0000

10.0000

.11 .9000

15.7000

18.8000

18.8000

22.2000

26.9000
32.6000

40.8000

19.5000

.0000

.000

.0000

.0000

.0000

.0000

.0000

.0000

.0000

.0000

.0000

8.8000

8.8000
14.2000

14.2000
19.9000

27.3000

37.9000

.9000
.9000
$M(5)$

19

807 ID $3 / 16$

.9125
-1.2151

-1.2151
-1.1087

90.7290

2.0000

2.0000

3.2000

5.1000

8.9000

14.9000

27.0000

.0000

.0000

.0000

.0000

.0000

.0000

0000

.0000

.0000

.0000

.0000

.5000

1.0000

1. 3000

5.3000

0.0000

8.6000

14.1000
25.8000

25.8000
1.0000

.0000
.0000

.0000

.0000

.0000

.0000

.0000

.0000

.0000

.3000

.7000
1.1000 


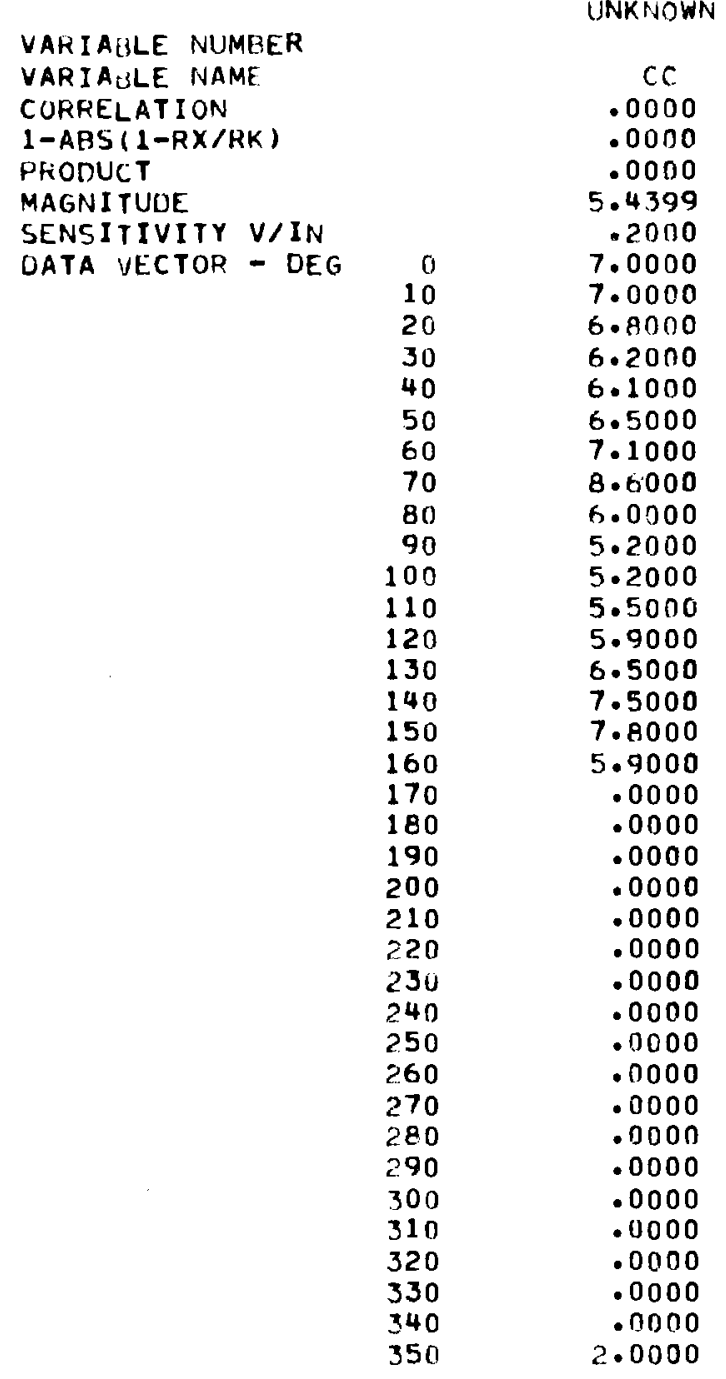

$M(1)$
6
$207001 / 16$
1.0000
.9997
.9996
5.4415
.2000
7.0000
7.0000
6.7000
6.3000
6.2000
6.4000
7.1000
8.6000
6.0000
5.3000
5.3000
5.5000
5.9000
6.5000
7.4000
7.8000
5.9000
.0000
.0000
.0000
.0000
.0000
.0000
.0000
.0000
.0000
.0000
.0000
.0000
.0000
.0000
.0000
.0000
.0000
.0000
2.0000

$M(2)$
18
$500.000 R$
.7690
.7234
.5568
7.5194
.2000
8.8000
9.0000
9.0000
8.4000
7.4000
6.8000
6.3000
6.0000
5.9000
5.9000
6.0000
6.1000
6.5000
7.0000
8.0000
9.1000
.0000
.0000
.0000
.0000
.0000
.0000
.0000
.0000
.0000
.0000
.0000
2.5000
7.8000
8.1000
8.3000
8.1000
8.1000
8.1000
8.5000
6.6000

$M(3)$
5
$201001 / 8$
.7903
.5576
.4407
3.7714
.2000
9.5000
3.2000
3.0000
2.4000
2.5000
2.9000
3.0000
3.2000
3.9000
2.9000
3.0000
3.0000
3.2005
3.0000
4.2000
5.1000
6.5000
.1000
.1000
.1000
.1000
.1000
.2000
.3000
.5000
.9000
1.0000
.0000
.0000
.0000
.0060
.0000
.0000
.0000
10.0000
4.5000

$M(4)$
10
$40 \% 001110$
.4260
.7367
.3143
7.3838
.2000
2.5000
2.3000
2.1000
2.0000
.0000
.0000
.0000
.0000
.0000
.0000
.0000
.0000
5.0000
7.5000
9.9000
12.4000
15.1000
15.8000
.0000
.0000
.0000
.0000
.0000
.0000
.0000
.0000
.0000
.0000
.0000
.0000
.0000
.0000
.0000
9.4000
14.9000
14.9000

$M(5)$
4
$209003 / 16$
.3012
.7190
.2599
7.5600
.20100
.0000
.0000
.0000
.0000
.0000
.0000
.0000
.0000
.0000
10.0000
1.4000
9.6000
10.0000
10.0000
11.0000
.0000
.0000
.0000
.0000
.0000
.0000
.0000
.0000
.0000
.0000
4.9000
10.9000
7.2000
8.7000
10.0000
11.2000
12.9000
7.5000
.0000
.0000
.0000




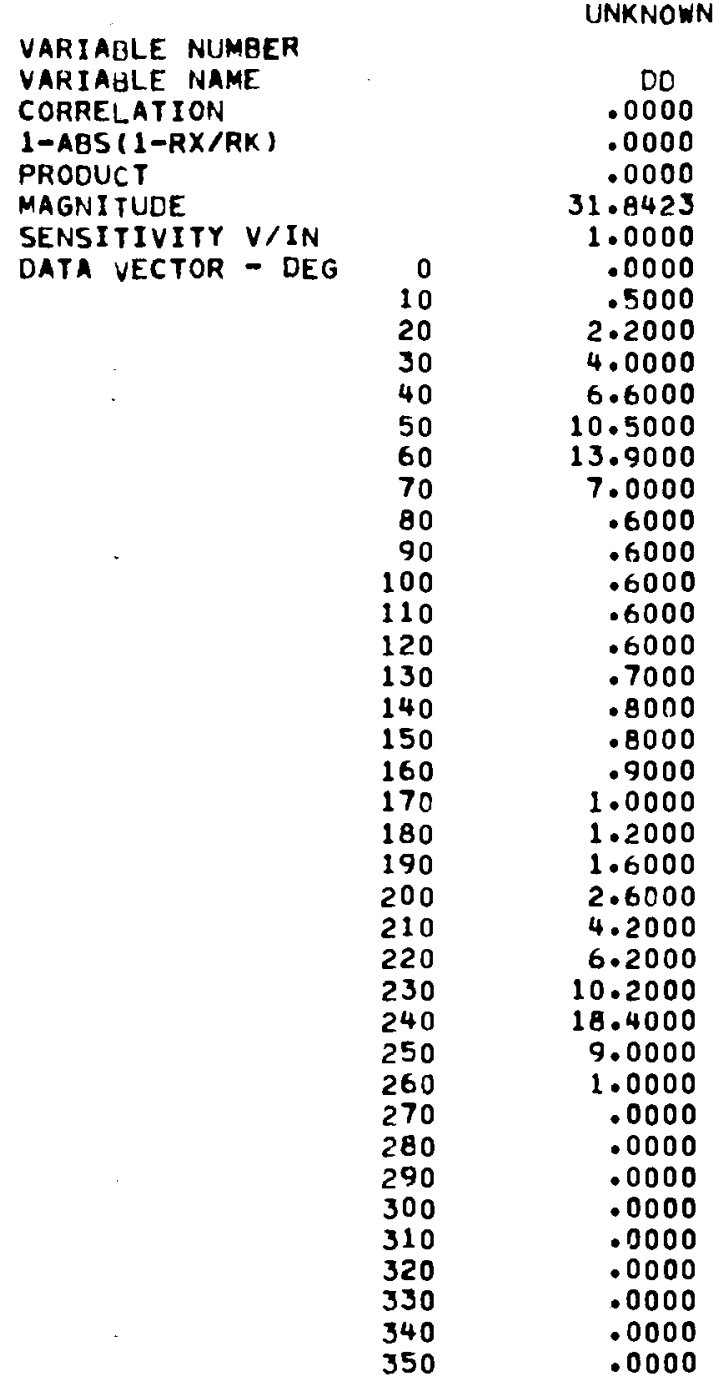

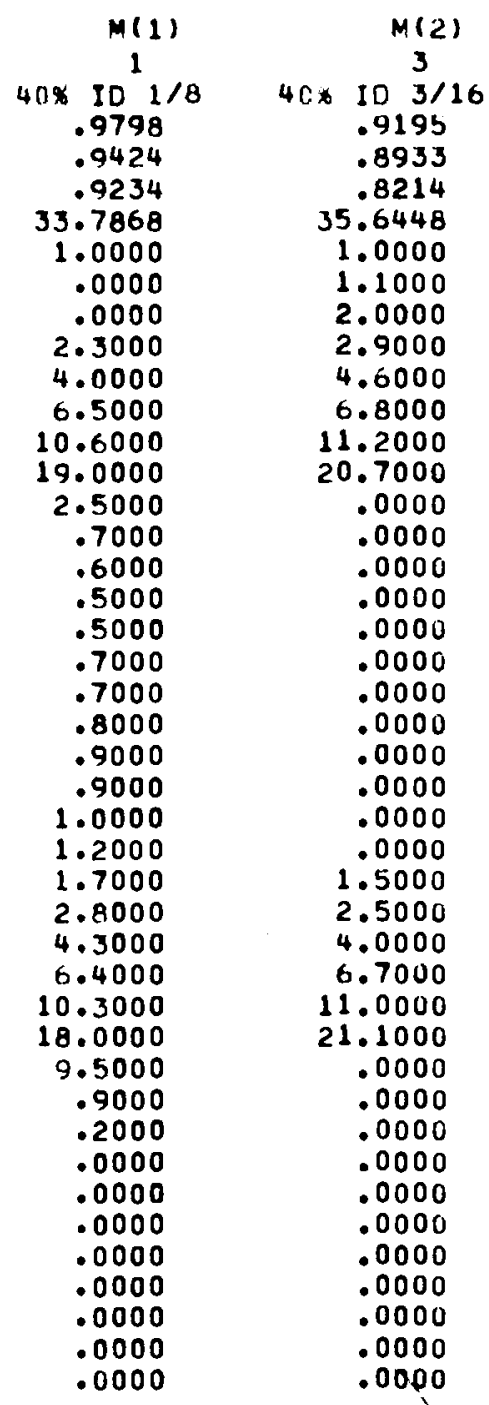

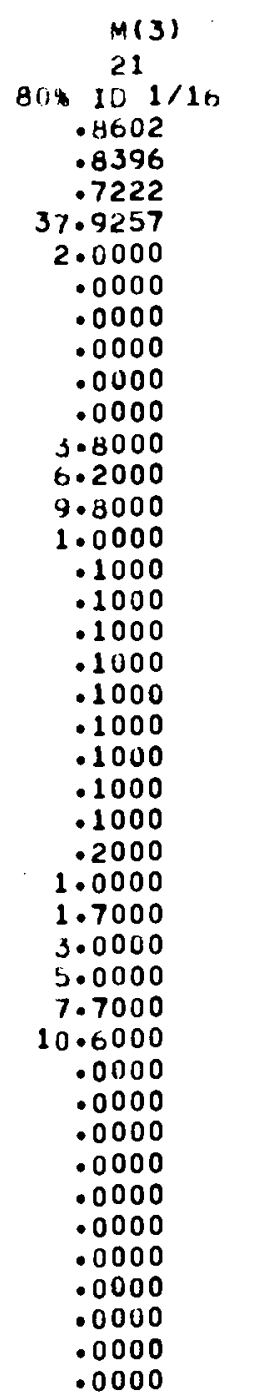

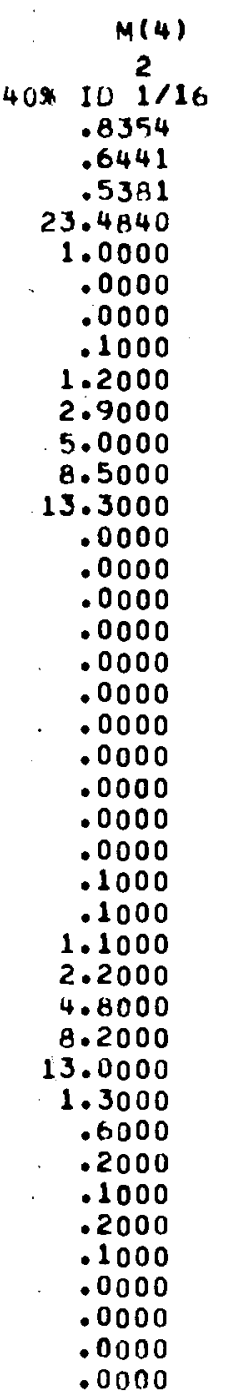

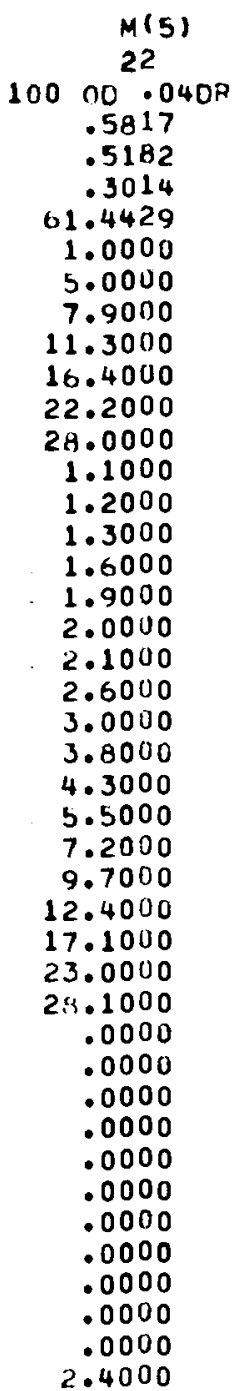


VARIAULE NUMBER

CORRELATION

1-ABS (1-RX/RK)

PRODUCT

MAGNI TUDE

SENSITIVITY VIIN

DATA VECTOR - DEG
UNKNOUN

.000

.0000

.0000

7.4857

$.20 n 0$

8.8000

9.0000

9.0000
8.5000

7.5000

6.8000

6.5000

6.0000

5.9000

5.9000

5.9000

6.1000

6.7000

6.0000

8.0000

9.1000

.0000
.0000

.0000

.0000

.0000

.0000

.0000

.0000

.0000

.0000

3.0000

7. 8000

8.1000

8.2000

8.0000

A. 1000

8.0000

A. 5000
$M(1)$

18

50 $00.020 R$

.9995

.9955

.9950
.5194

.2000

8.8000

9.0000

9.0000

8.4000

7.4000

6.8000

.3000

6.0000

5.9000

5.9000

6.0000

0.1000

6.6000

8.0000

8.0000
9.1000

9.1000
.0000

.0000

.0000

.0000

.0000

.0000

.0000

.0000

.0000

.0000

2.5000

7.8000

8.1000

3.3000

8.1000

8.1000

8.1000

8.5000

8.6000

14
$208003 / 16$
.5835
.9902
.5777
7.5600
.2000
.0000
.0000
.0000
.0000
.0000
.0000
.0000
.0000
.0000
10.0000
9.4000
9.6000
10.0000
10.8000
11.8000
.0000
.0000
.0000
.0000
.0000
.0000
.0000
.0000
.0000
.0000
9.9000
10.9000
7.2000
8.7000
10.0000
11.2000
12.9000
7.5000
.0000
.0000
.0000

113
10
$40 \% 1116$
.4893
.9862
.4826
7.3030
.2000
2.5000
2.3000
2.1000
2.0000
.0000
.0000
.0000
.0000
.0000
.0000
.0000
.0000
5.0000
7.5000
9.9000
12.4000
15.1000
15.8000
.0000
.0000
.0000
.0000
.0000
.0000
.0000
.0000
.0000
.0000
.0000
.6000
.0000
.0000
.0000
. .4000
14.9000
14.4000

$m(4)$
$2061 / 16$
.7693
.6243
.4803
5.4415
.2000
7.0000
7.0000
6.7000
6.3000
6.2000
6.4000
7.1000
8.6000
6.0000
5.3000
5.3000
5.5000
5.9000
6.5000
7.4000
7.8000
5.9000
.0000
.0005
.0000
.0000
.0000
.0000
.0000
.0000
.0000
.0000
.0000
.0000
.0000
.0000
.0000
.0000
.0000
.0000
2.0000

$17(5)$
16
800.0201
.6030
.7050
.4251
10.6184
.2000
17.6000
21.1000
1.0000
.4000
.0000
.0000
.0000
.0000
.0000
.0000
.9000
3.5000
5.40110
7.8000
4.3000
11.2000
13.0000
14.9000
17.1000
19.2000
1.8000
.6000
.0000
.0000
.0000
.0000
.0000
.0000
.0000
7.0000
7.2000
7.9000
8.8000
10.00100
12.0000
14.20110
4




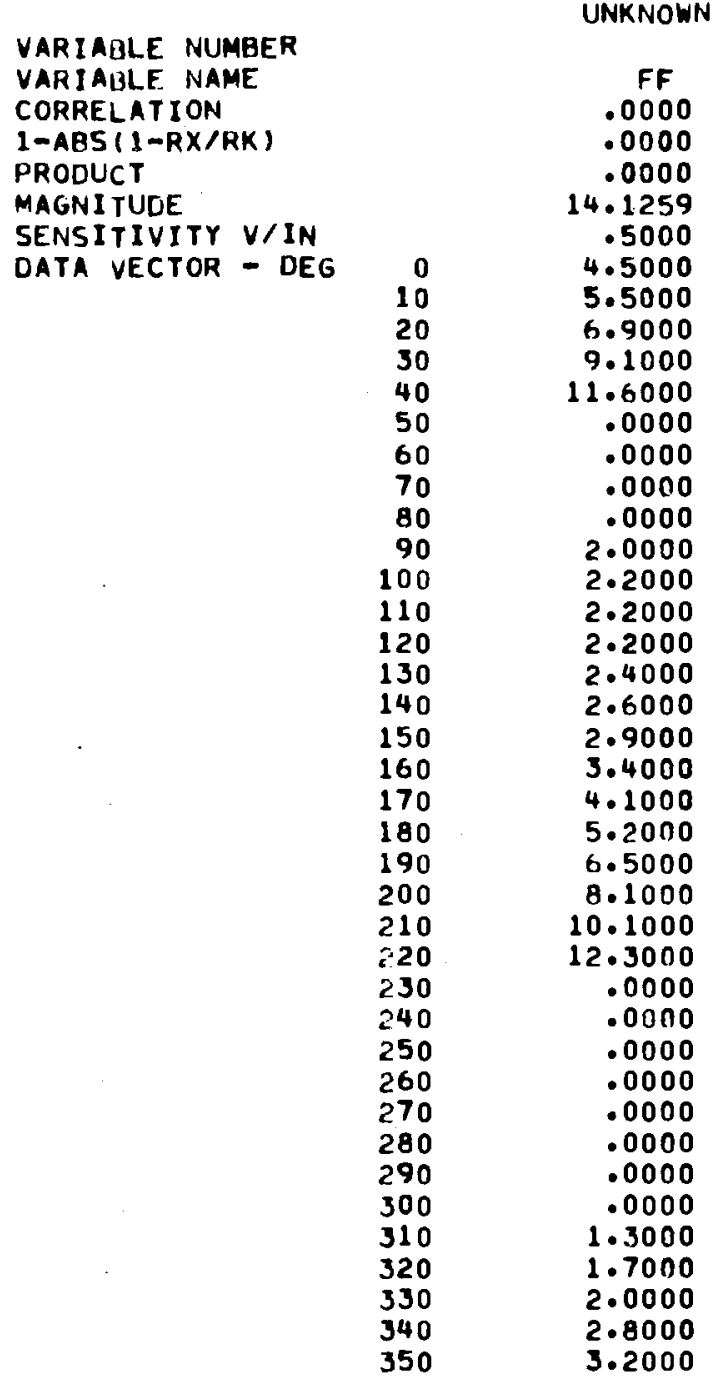

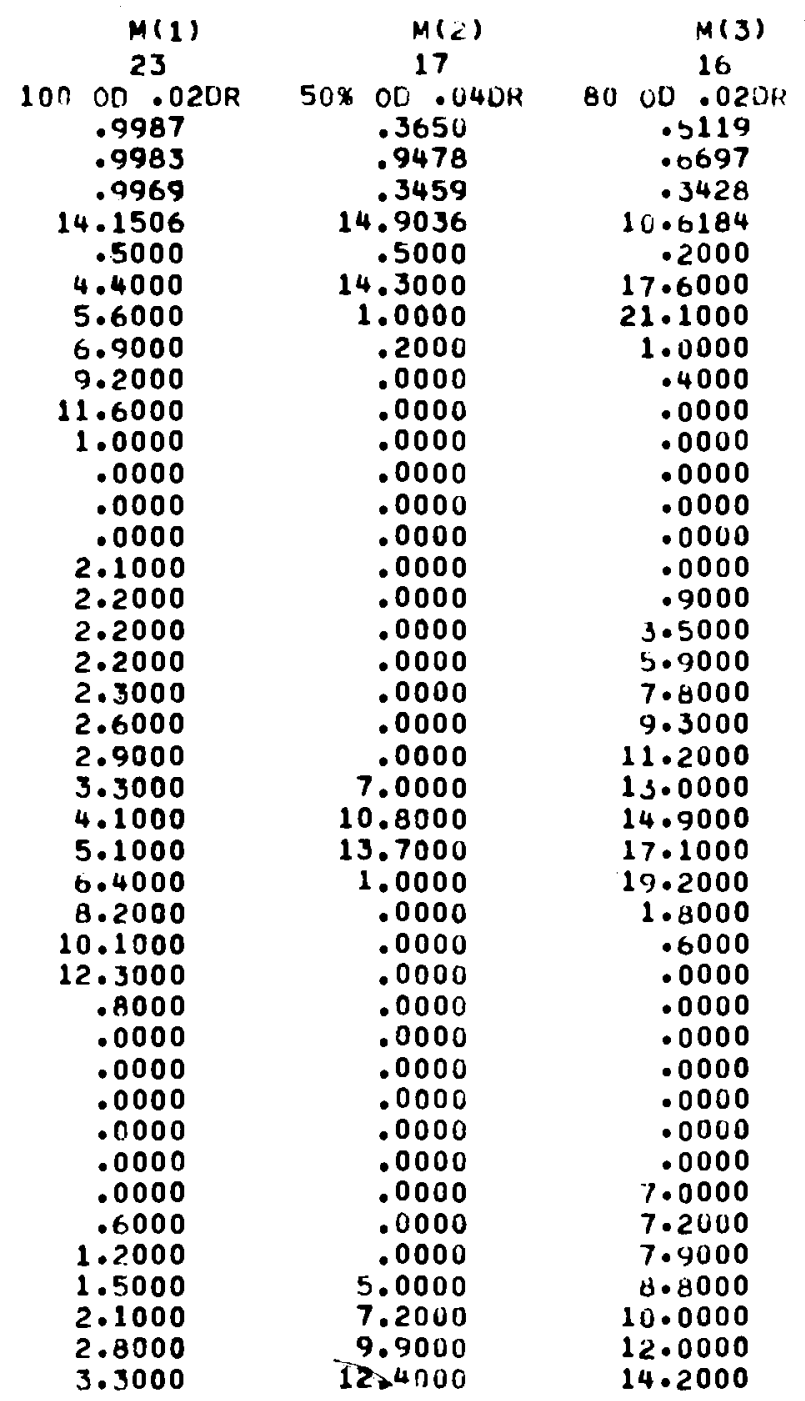

$M(4)$
12
$40 \% 101 / 8$
.2748
.8904
.2447
15.8639
.2000
.9000
.9000
.1000
.1000
.1000
.1000
.2000
.2000
.2000
.3000
.3000
.6000
1.0000
2.0000
7.3000
14.9000
24.1000
34.1000
.0000
.0000
.0000
.0000
.0000
.0000
.0000
.0000
.0000
6.5000
9.3000
12.0000
15.3000
19.4000
24.0000
29.1000
33.7000
28.7000
$M(5)$




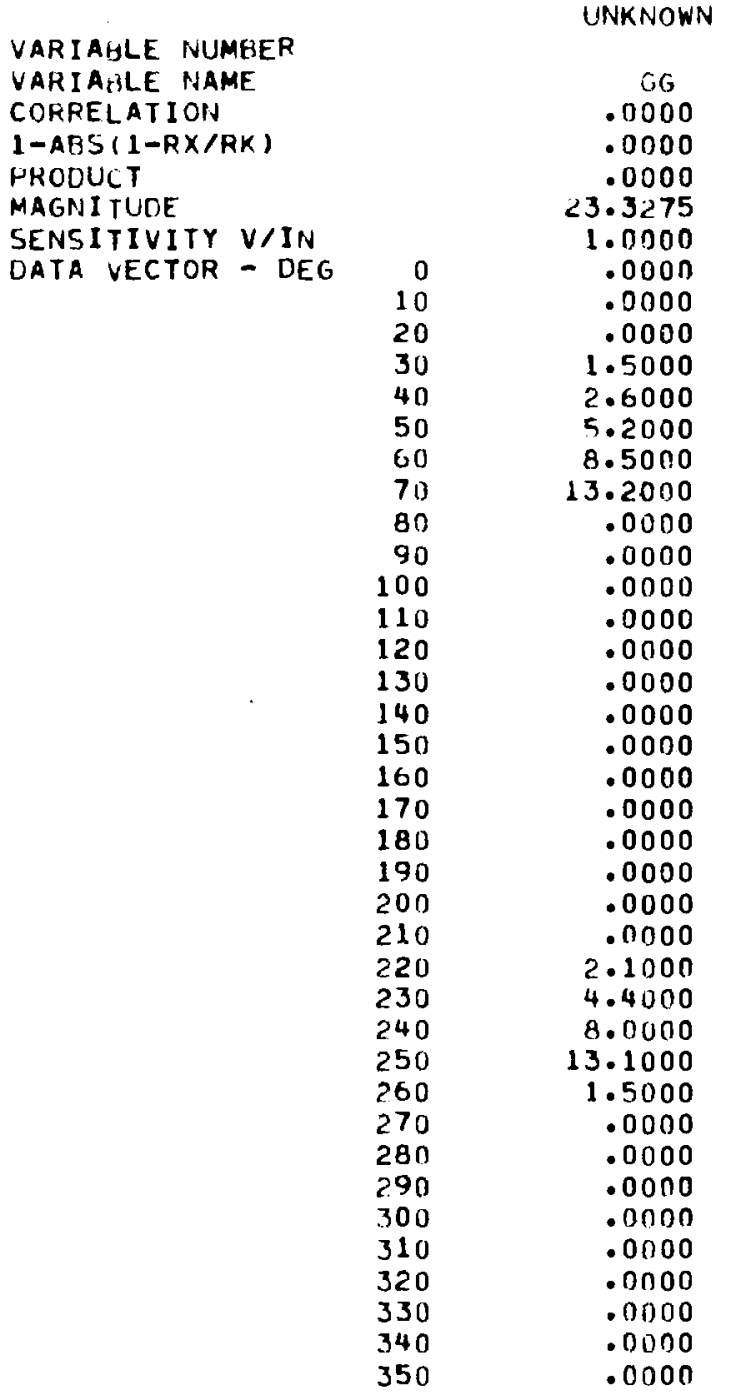

\begin{tabular}{|c|c|c|}
\hline & $M(1)$ & \\
\hline & $2^{2} 16$ & $2088^{8}$ \\
\hline $40 \%$ & ID $1 / 16$ & $20 \times I 01 / 8$ \\
\hline & .09833 & $\begin{array}{r}.9591 \\
.7794\end{array}$ \\
\hline & .9914 & .7475 \\
\hline & 3.4840 & 19.1114 \\
\hline & .0000 & .5000 \\
\hline & .0000 & .5000 \\
\hline & .0000 & .6000 \\
\hline & .1000 & .8000 \\
\hline & .2000 & .9000 \\
\hline & .9000 & 1.2000 \\
\hline & .0000 & 4.5000 \\
\hline & .5000 & 11.0000 \\
\hline & .3000 & 22.0006 \\
\hline & .0000 & .0000 \\
\hline & .0000 & .0000 \\
\hline & .0000 & 1.5000 \\
\hline & .0000 & 1.0000 \\
\hline & .0000 & 1.8000 \\
\hline & .0000 & 1.8000 \\
\hline & .0000 & 1.8000 \\
\hline & .0000 & 1.9000 \\
\hline & .0000 & 2.0000 \\
\hline & .0000 & 2.1000 \\
\hline & .0000 & 2.4000 \\
\hline & .1000 & 2.8000 \\
\hline & .1000 & 3.2000 \\
\hline & .1000 & 3.8000 \\
\hline & .2000 & 5.5000 \\
\hline & .8000 & 7.9000 \\
\hline & .2000 & 12.3000 \\
\hline & 3.0000 & 22.8000 \\
\hline & .3000 & .0000 \\
\hline & .6000 & 1.2000 \\
\hline & .2000 & .7000 \\
\hline & .1000 & .5000 \\
\hline & .2000 & .4000 \\
\hline & .1000 & .4000 \\
\hline & .0000 & .3000 \\
\hline & .0000 & . $30 \cup 0$ \\
\hline & .0000 & .3000 \\
\hline & .0000 & .4000 \\
\hline
\end{tabular}

$M(3)$
707
105311
.9745
.5582
.5439
10.1793
.5000
2.0000
2.2000
2.6000
3.1000
4.1000
6.0000
9.4000
11.4000
.2000
.2000
.2000
.3000
.3000
.3000
.4000
.5000
.5000
.6000
.8000
.9000
1.1000
1.3000
3.3000
5.4000
4.8000
18.5000
1.1000
1.1000
1.2000
1.2000
1.3000
1.3000
1.4000
1.5000
1.0000
1.4000

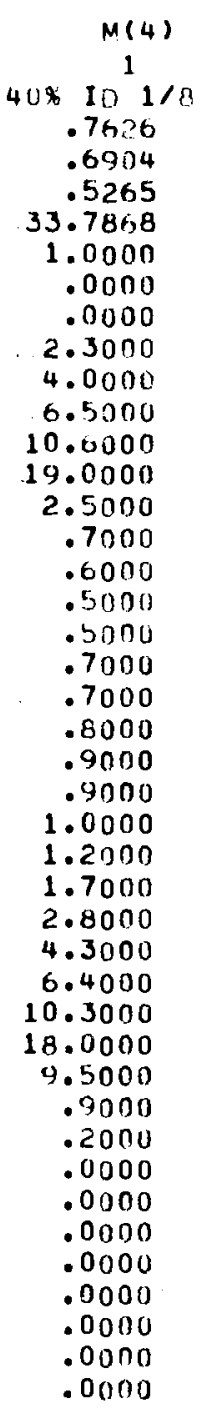

$19(5)$
21
$80 \% .1171 / 16$
.7481
.6151
.4602
37.9257
2.0000
.0000
.0000
.0000
.0000
.0000
3.8000
6.2000
9.8000
1.0000
.1000
.1000
.1000
.1000
.1000
.1000
.1000
.1000
.1000
.2000
1.0000
1.7000
3.0000
5.0000
7.1000
10.6000
.0000
.0000
.0000
.0000
.0000
.0000
.0000
.0000
.0000
.0000
.0000


VARIAGLE NUMBER VARIAULE NAME CORRELATION

1-ABS $(1-R X / R K)$

PRODUCT

MAGNITUDE

SENSITIVITY $V / I N$

DATA VECTOR - DEG
UNK NOWN

$\mathrm{HH}$
.0000

.0000

.0000

35.6758

2.0000

.0000

.0000

.0000

1.5000

3.9000

6.4000

7.2000
1.0000

1.0000
.0000

.0000
.0000

.0000

.0000

.0000

.0000

.0000

.0000

.0000
.0000

.5000

1.6000

3.0000

5.0000

7.6000

10.6000
.0000

.0000
.0000

.0000

.0000
.0000

.0000

.0000

.0000

.0000

.0000

.0000
.0000
$M(1)$

21

80x ID $1 / 16$

.9879
.9407

.9293

37.9257

2.0000

2.0000
.0000

.0000
.0000

.0000

.0000

.0000

3.8000

6.2000

9.8000
1.0000

.1000

.1000

.1000

.1000

.1000

.1000

.1000

.1000
.1000

.2000

1.0000

1.7000

3.0000

5.0000

10.6000

.0000

.0000

.0000

.0000

.0000

.0000

.0000

.0000

.0000
.0000

$11(2)$
3
$103 / 16$
.8554
.9991
.8546
35.6448
1.0000
1.1000
2.0000
2.9000
4.6000
6.8000
11.2000
20.7000
.0000
.0000
.0000
.0000
.0000
.0000
.0000
.0000
.0000
.0000
.0000
.0000
1.5000
2.5000
4.0000
6.7000
11.0000
21.1000
.0000
.0000
.0000
.0000
.0000
.0000
.0000
.0000
.0000
.0000
.0000

3

1
$413 \% 10118$
.8474
.9441
.8000
33.7868
1.0000
.0000
.0000
2.3000
4.0000
0.5000

6.5000

10.0000

19.0000
2.5000

.7000

.6000

.5000
.5000

.500

.7000

.8000

.8000

.9000

1.0000

1.2000

1.7000

2.8000

4.3000

10.3000

10.30000

9.5000

.9000

.2000
.0000

.0000

.0000

.0000

.0000

.0000

.0000
.0000

$M(4)$
2
$40 \times 101 / 10$
.7446
.4809
.3580
23.4540
1.0000
.0000
.0000
.1000
1.2000
2.9000
5.0000
8.5000
13.3000
.0000
.0000
.0000
.0000
.0000
.0000
.0000
.0000
.0000
.0000
.0000
.1000
.1000
1.1000
2.2000
4.8000
8.2000
13.0000
1.3000
.6000
.2000
.1000
.2000
.1000
.0000
.0000
.0000
.0000

$M(5)$
22
$10000.040 R$
.5146
.5806
.2988
61.4429
1.0000
5.0000
7.9000
11.3000
16.4000
22.2000
28.0000
1.1000
1.2000
1.3000
1.6000
1.9000
2.0000
2.1000
2.6000
3.0000
3.8000
4.3000
5.5000
7.2000
9.7000
12.4000
17.1000
23.0000
28.1000
.0000
.0000
.0000
.0000
.0000
.0000
.0000
.0000
.0000
.0000
.0000
2.4000

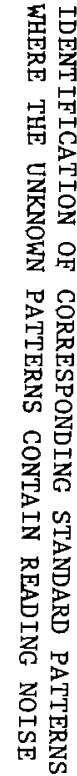




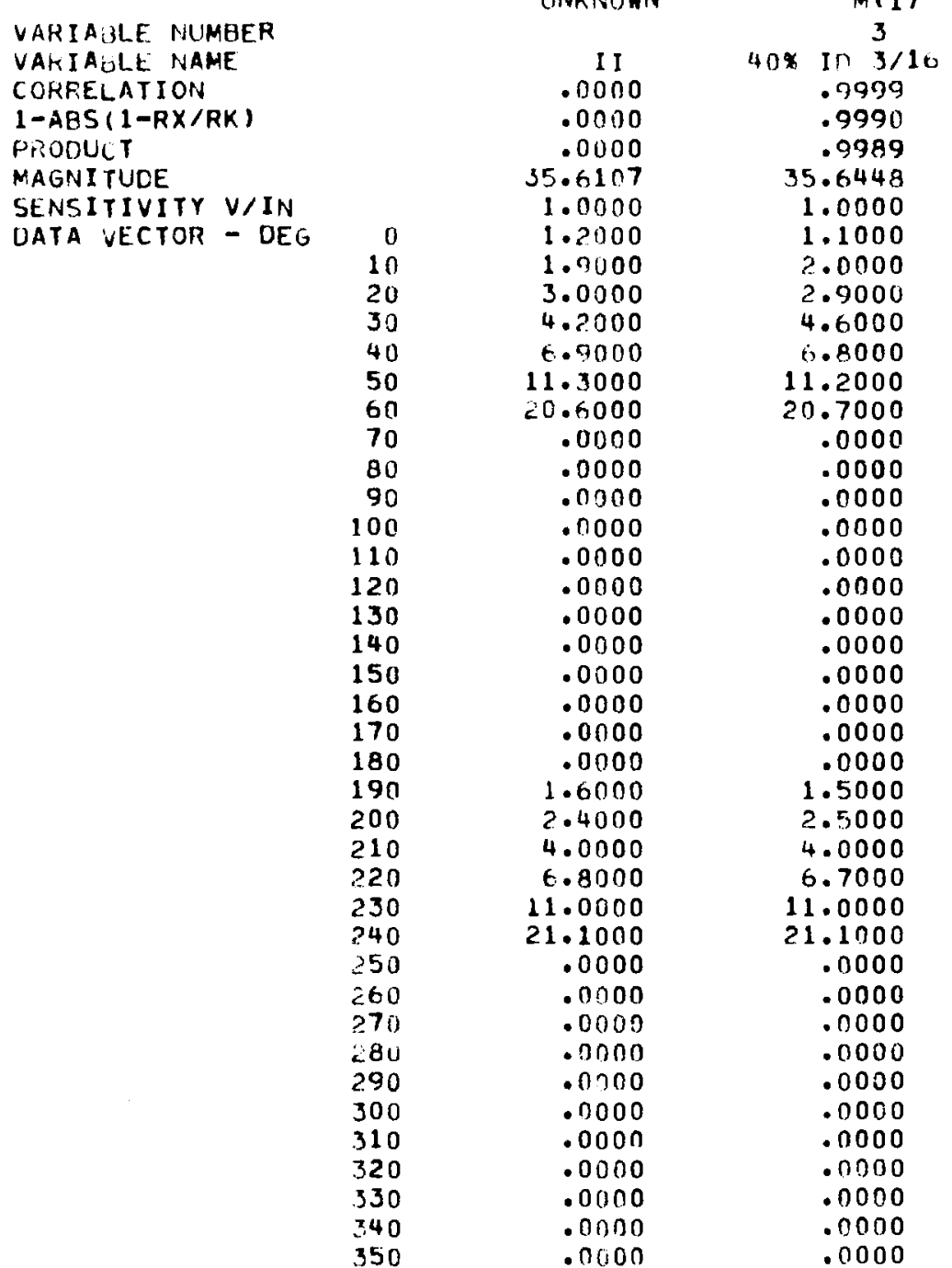

$M(2)$
1
$40 \times 101$
.9500
.9400
.8907
33.7806
1.0000
.0000
.0000
2.3000
4.0000
6.5000
10.6000
19.0000
2.5000
.7000
.6000
.5000
.5000
.7000
.7000
.8000
.9000
.9000
1.0000
1.2000
1.7000
2.8000
4.3000
6.4000
10.3000
18.0000
9.5000
.9000
.2000
.0000
.0000
.0000
.0000
.0000
.0000
.0000
.0000

1
2131
80151116
.7059
.9390
.7579
37.9257
2.0000
.0000
.0000
.0000
.0000
.0000
3.0000
6.2000
9.3000
1.0000
.1000
.1000
.1000
.1000
.1000
.1000
.1000
.1000
.1000
.2000
1.0000
1.7000
3.0000
5.0000
7.7000
10.0000
.0000
.0000
.0000
.0000
.0000
.0000
.0000
.0000
.0000
.0000
.0000

$29(4)$
22
100.00 .0421
.52163
.5790
.3106
01.44 .29
1.0000
5.0000
7.9000
11.3000
16.4000
22.2000
28.0000
1.1000
1.2000
1.3000
1.0000
1.9000
2.0000
2.1000
2.6000
3.0000
3.8000
4.3000
5.5000
7.2000
9.7000
12.4000
17.1000
23.0000
28.1000
.0000
.0000
.0000
.0000
.0000
.0000
.0000
.0000
.0000
.0000
.0000
2.4000

$N(5)$
211
1011
.7531
.1010
.3020
$8 \% .3041$
2.0000
.0000
2.0000
4.0000
6.9000
11.0000
16.4000
20.5000
.0000
.0000
.0000
.0000
.0000
.0000
.0000
.0000
.0000
.5000
1.0000
1.9000
4.0000
0.0000
10.1000
17.4000
23.5000
.0000
.0000
.0000
.0000
.0000
.0000
.0000
.0000
.0000
.0000
.0000
.00100

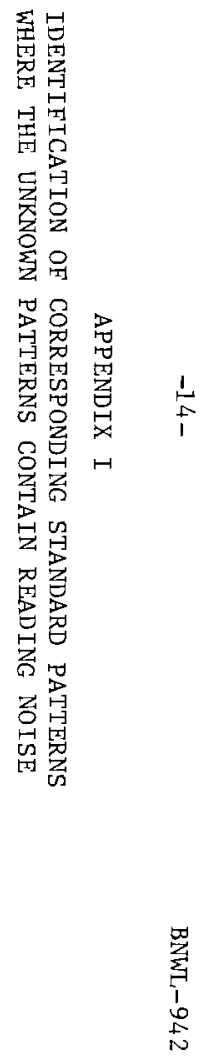


VAKIABLE NUMBER VARIABLE NAME

CORPELATION

1-ABS $(1-R X / R K)$

PRODUCT

DATA VECTOR - DEG

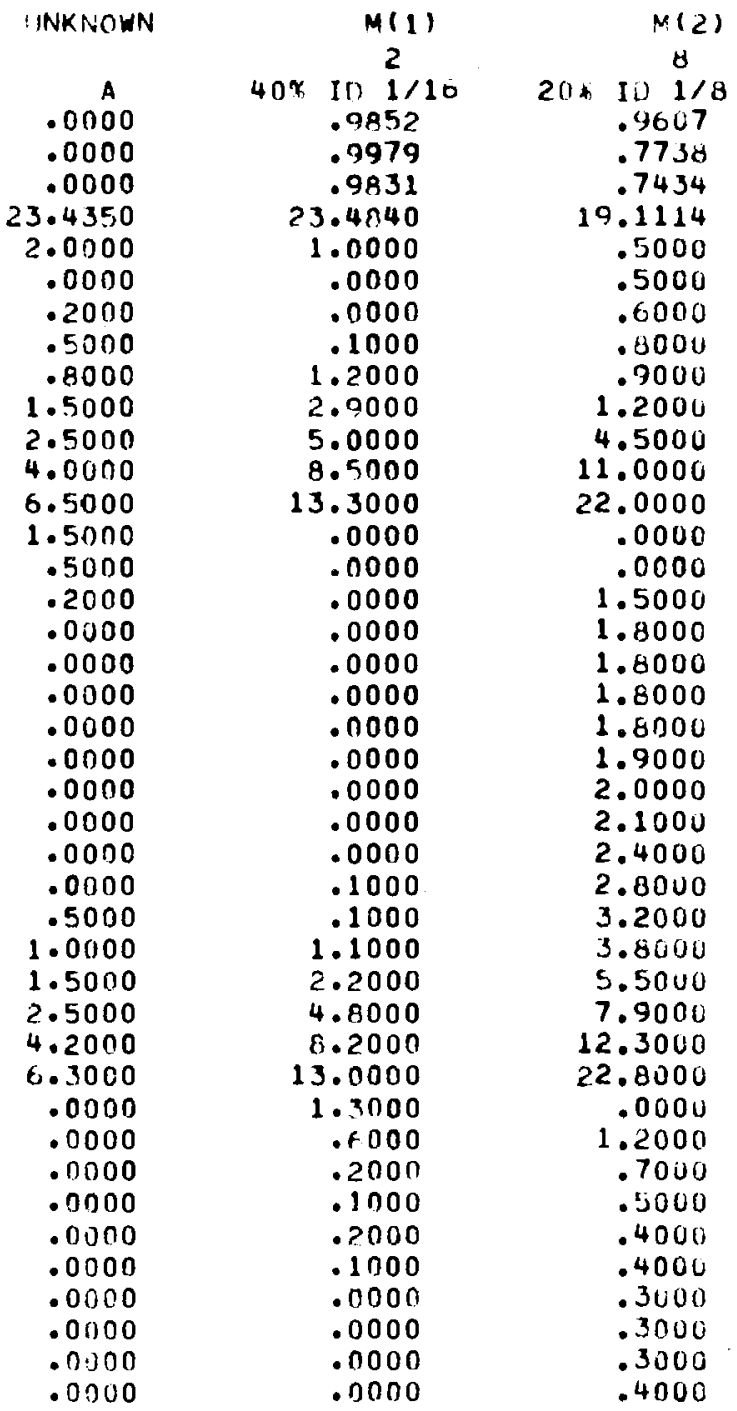

$M 131$
1
40.1118
.7451
.6930
.5445
33.7868
1.0000
.0000
.0000
2.3000
4.0000
6.5000
10.6000
19.0000
2.5000
.7000
.6000
.5000
.5000
.7000
.7000
.2000
.9000
.9000
1.0000
1.2000
1.7000
2.0000
4.3000
6.4000
10.3000
10.0000
4.5000
.3000
.2000
.0000
.0000
.0000
.0000
.0000
.15000
.0000
.0000

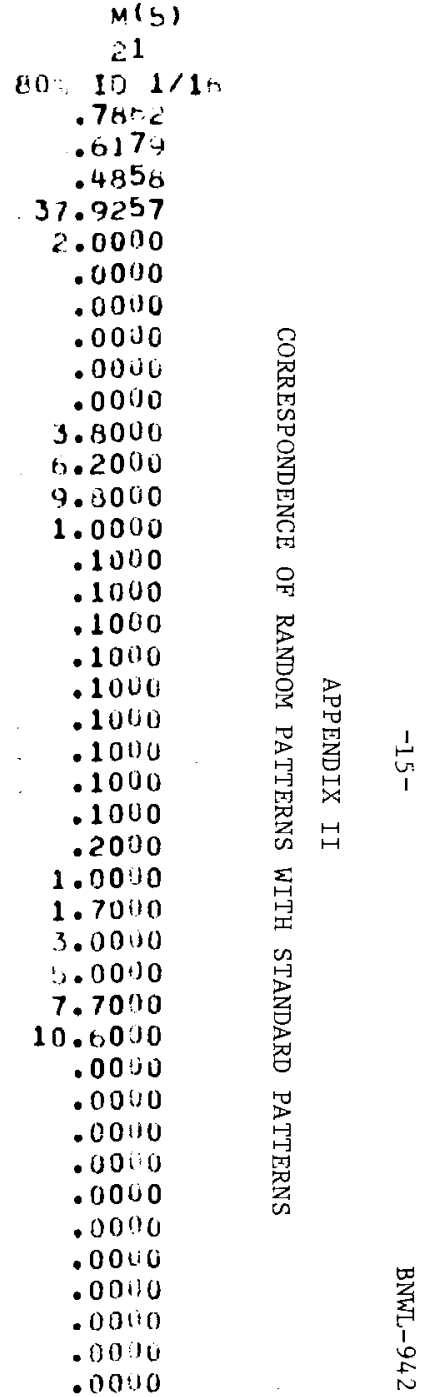


VARIAGLE NUMBER

VARI ABLE NAME

CORRELATION

1-ABS (1-RX/RK)

PRODUCT

MAGNITUDE

SENSITIVITY V/IN

DATA VECTOR - DEG

\begin{tabular}{|c|c|c|}
\hline UNKNOWN & $M(1)$ & $M(2)$ \\
\hline B & $40 \times$ io $3 / 10$ & $40 \times$ ID $1 / 8$ \\
\hline .0000 & .8993 & .9605 \\
\hline .0000 & .8961 & .8354 \\
\hline .0000 & .8058 & .8024 \\
\hline $\begin{array}{r}39.3477 \\
2.0000\end{array}$ & 35.6448 & 33.7868 \\
\hline $\begin{array}{l}2.0000 \\
1.6000\end{array}$ & 1.0000 & 1.0000 \\
\hline $\begin{array}{l}1.6000 \\
1.9000\end{array}$ & 1.1000 & .0000 \\
\hline $\begin{array}{l}1.9000 \\
2.3000\end{array}$ & 2.0000 & .0000 \\
\hline $\begin{array}{l}2.3000 \\
2.8000\end{array}$ & $\begin{array}{l}2.9000 \\
4.6000\end{array}$ & 2.3000 \\
\hline $\begin{array}{l}2.8000 \\
4.2000\end{array}$ & 6.8000 & 6.5000 \\
\hline 6.2000 & 11.2000 & 10.6000 \\
\hline $10 \cdot 3000$ & 20.7000 & 19.0000 \\
\hline .0000 & .0000 & 2.5000 \\
\hline .0000 & .0000 & .7000 \\
\hline $\begin{array}{l}.0000 \\
.0000\end{array}$ & $\begin{array}{l}.0000 \\
.0000\end{array}$ & .6000 \\
\hline .0000 & .0000 & .5000 \\
\hline .0000 & .0000 & .7000 \\
\hline .0000 & .0000 & .7000 \\
\hline .0000 & .0000 & .8000 \\
\hline $\begin{array}{l}.0000 \\
.0000\end{array}$ & .0000 & .9000 \\
\hline .0000 & .0000 & .9000 \\
\hline .0000 & .0000 & 1.2000 \\
\hline .0000 & 1.5000 & 1.7000 \\
\hline .0000 & 2.5000 & 2.8000 \\
\hline 1.5000 & 4.0000 & 4.3000 \\
\hline 3.0000 & 6.7000 & 6.4000 \\
\hline $5 \cdot 2000$ & 11.0000 & 10.3000 \\
\hline 0.0000 & 21.1000 & 18.0000 \\
\hline 7.7000 & .0000 & 9.5000 \\
\hline .9000 & .0000 & .9000 \\
\hline .5000 & .0000 & .2000 \\
\hline .5000 & .0000 & .0000 \\
\hline .6000 & $\begin{array}{l}.0000 \\
.0000\end{array}$ & $\begin{array}{r}.0000 \\
.0000\end{array}$ \\
\hline .7000 & $\begin{array}{l}.0000 \\
.0000\end{array}$ & .0000 \\
\hline $\begin{array}{r}.8000 \\
.9000\end{array}$ & $\begin{array}{l}.0000 \\
.0000\end{array}$ & .0000 \\
\hline 1.0000 & .0000 & .0000 \\
\hline 1.2000 & .0000 & .0000 \\
\hline 1.4000 & .0000 & .0000 \\
\hline
\end{tabular}

$M(4)$
22
$10000.040 R$
.5106
.6404
.3270
61.4429
1.0000
5.0000
7.9000
11.3000
16.4000
22.2000
28.0000
1.1000
1.2000
1.3000
1.6000
1.9000
2.0000
2.1000
2.6000
3.0000
3.8000
4.3000
.5 .5000
7.2000
9.7000
12.4000
17.1000
23.0000
28.1000
.0000
.0000
.0000
.0000
.0000
.0000
.0000
.0000
.0000
.0000
.0000
2.4000

2.4000

$M(3)$
21
$80 \% 101 / 16$
.6780
.9625
.6526
37.9257
2.0000
.0000
.0000
.0000
.0000
.0000
3.8000
6.2000
9.8000
1.0000
.1000
.1000
.1000
.1000
.1000
.1000
.1000
.1000
.1000
.2000
1.0000
1.7000
3.0000
5.0000
7.7000
10.6000
.0000
.0000
.0000
.0000
.0000
.0000
.0000
.0000
.0000
.0000
.0000


VARIAISLE NUMIJER

VARI IALLE NAME

CORPELATION

1-ABS (1-RX/RK)

PRODUCT

MAGNITUDE

SENSITIVITY V/IN

OATA VECTOR - DEG
UNKNOWH

\begin{tabular}{|c|c|c|}
\hline & UNKNOWH & $4(1)$ \\
\hline & c & $4 n x$ in $3 / 10$ \\
\hline & .0000 & .0950 \\
\hline & .0000 & $.8 B 70$ \\
\hline & .0000 & .8826 \\
\hline & 39.6717 & 35.6448 \\
\hline & 2.0000 & 1.0000 \\
\hline 0 & .0000 & 1.1000 \\
\hline 10 & 1.0000 & 2.0000 \\
\hline 20 & 1.5000 & 2.9000 \\
\hline 30 & 2.0000 & 4.6000 \\
\hline 40 & 3.5000 & 6.8000 \\
\hline 50 & 5.8000 & 11.2000 \\
\hline 60 & 11.3000 & 20.7000 \\
\hline 70 & .0000 & .0000 \\
\hline 80 & .0000 & .0000 \\
\hline 90 & .0000 & .0000 \\
\hline 100 & .0000 & .0000 \\
\hline 110 & .1000 & .0000 \\
\hline 120 & .2000 & .0000 \\
\hline 130 & .3000 & .0000 \\
\hline 140 & .4000 & .0000 \\
\hline 150 & .5000 & .0000 \\
\hline 160 & .6000 & .0000 \\
\hline 170 & .7000 & .0000 \\
\hline 180 & 1.0000 & .0000 \\
\hline 190 & 1.3000 & 1.5000 \\
\hline 200 & 1.7000 & 2.5000 \\
\hline 210 & 2.5000 & 4.0000 \\
\hline 220 & 3.8000 & 0.7000 \\
\hline 230 & 6.4000 & 11.0000 \\
\hline 240 & 12.0000 & 21.1000 \\
\hline 250 & .0000 & .0000 \\
\hline 260 & .0000 & .0000 \\
\hline 270 & .0000 & . กnoo \\
\hline 280 & .0000 & .0000 \\
\hline 290 & $.00 \cap 0$ & .0000 \\
\hline 300 & .0000 & .0000 \\
\hline 310 & .0000 & . \\
\hline 320 & .0000 & .0000 \\
\hline 330 & .0000 & .0000 \\
\hline 340 & .0000 & .0000 \\
\hline 350 & .0000 & .0000 \\
\hline
\end{tabular}

Ne

$40 \% 10^{1} 1 / 8$

.0519

.8258
.7861

.7861
3.7868

1.0000

.0000

.0000

2.3000

4.0000
6.5000

6.5000
10.6000

19.0000

2.5000

.7000

.6000

.5000
.5000

. .5000

.7000

.8000

.9000

.9000

1.0000

1.2000

1.7000

2. 8000

4.3000

6. 4000

10.3000

18.0000

9.5000

.3000

.2000
.0000

.0000

.0000

.0000

.0000

.0000

.0000
He. $\frac{1}{101 / 16}$

.7909

.3540
.7002

.7002
$3 \% .4257$

2.0000

.0000

.0000

.0000

.0000

3. 8000

0.2000

1.0000

.1000

.1000

- 1000

.1000

.11000

- 1000

.1000

.1000

.2000
1.0000

1.0000
1.7000

3.0000

5.0000

7.7000

$10 \cdot n 000$

$-0000$

.0000

. 0000

.0000

- j000

.0000

- 0000

- 0000

.0000 $\because(4)$

(14) $00^{22}$

.5514
.0457
.3560
1.4429
1.0000

1.0000

5.00ก0

7.9000

11.3000
16.4000

16.4000
22.2000

28.0000

1.1000

1.2000

1.3000

1.6000

1.9000
2.0000

2.0000

2.0000

3.0000

$3 . \$ 10 n 0$

4.3000

5.3000

9.7003

12.4000

17.1000

23.0000

23.1000

- 0000

.0000

.0000

.0000

.0000

. 0000

.0000

- anno

- onna

2.4000 
VARIABLE NUMBER

CORRELATION

1-ABS $(1-R X / R K)$

PRODUCT

MAGN I TUDE

SENSITIVITY V/IN

DATA VECTOR - DEG
UNKNOWN

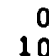

0
10
20

10
.0000
.0000
.0000
9.5462
1.0000
.0000
.0000
.0000
.0000
.0000
.0000
2.8000
4.5000
3.0000
1.0000
.8000
.7000
.6000
.5000
.5000
.5000
.5000
.5000
.5000
.6000
.7000
.8000
1.0000
1.6000
3.0000
6.0000
.0000
.0000
.0000
.0000
.0000
.0000
.0000
.0000
.0000
.0000

$40 \times 101 / 16$

.9070

.9065

.8222
0.5310

.5000

.5000

.1000

.1000

.1000

.2000

.2000

10.0000

10.0000

4.0000

3.0000
2.5000

2.1000

1.9000

1.8000

1.7000

1.7000

1.8000

1.9000

2.1000

2.4000

3.0000

4.0000

6.0000

10.0000

3.5000

.9000
.1000

.1000

.1000

.1000

.1000

.1000

.1000
.1000
$M(2)$

$M(2)$
77
$103 / 16$
.8793
.5900
.5188
16.1793
.5000
2.0000
2.2000
2.6000
3.1000
4.1000
6.0000
9.4000
19.4000
.2000
.2000
.2000
.3000
.3000
.3000
.4000
.5000
.5000
.6000
.8000
.9000
1.1000
1.8000
3.3000
5.4000
9.8000
18.5000
1.1000
1.1000
1.2000
1.2000
1.3000
1.3000
1.4000
1.5000
1.6000
1.8000

$M(4)$
2
$40 \times 101 / 16$
.8794
.4065
.3575
23.4840
1.0000
.0000
.0000
.1000
1.2000
2.9000
5.0000
8.5000
13.3000
.0000
.0000
.0000
.0000
.0000
.0000
.0000
.0000
.0000
.0000
.0000
.1000
.1000
1.1000
2.2000
4.8000
8.2000
13.0000
1.3000
.6000
.2000
.1000
.2000
11000
.0000
.0000
.0000
.0000

2

8

$101 / 8$
.9267

.4995

19.1114

.5000

.5000

.6000

.9000

1. 2000

4. 5000

22.0000

.0000

1.5000

1.8000

1. 8000

1.8000

1.3000

2.0000

2.1000

2.4000

2.8000

3. 2000

5.5000

7.9000

12.3000

2.8000

1. 2000

.7000

.5000

.4000

.4000

.3000

.3000
.4000

$M(5)$
4
$003 / 16$
.2797
.7373
.2062
7.5600
.2000
.0000
.0000
.0000
.0000
.0000
.0000
.0000
.0000
.0000
10.0000
9.4000
9.6000
10.0000
10.8000
11.8000
.0000
.0000
.0000
.0000
.0000
.0000
.0000
.0000
.0000
.0000
9.9000
10.9000
7.2000
8.7000
10.0000
11.2000
12.9000
7.5000
.0000
.0000
.0000


VAFIATLE: NUMTIER

VAKIAISLE NAME

COFRELATION!

$1-\operatorname{ABS}(1-R X / R K)$

FRODUCT

SENSI TIVITY VIN

LATA VECTOS - DEG

1 INKNONN
.0000
.0000
.0000
21.0858
1.0000
.5000
.5000
.5000
.5000
.6000
.9000
6.5000
13.7000
.0000
1.9000
1.7000
1.6000
1.5000
1.4000
1.3000
1.2000
1.3000
1.4000
1.5000
1.6000
1.8000
2.0000
6.4000
3.9000
6.0000
11.0000
.8000
.0000
.0000
.0000
.0000
.0000
.0000
.0000
.0000
.5000

$M(2)$
2
40.1110
.3325
.8979
.3423
23.4945
1.0000
.0000
.0000
.1000
1.2000
2.9000
5.0000
8.5000
13.3000
.0000
.0000
.0001
.0000
.0000
.0000
.0000
.0000
.0000
.0000
.0000
.1000
.1000
1.1000
2.2000
4.0000
.2000
13.0000
1.3000
.0000
.2000
.1000
.2000
.1000
.0000
.0000
.0000
.0000

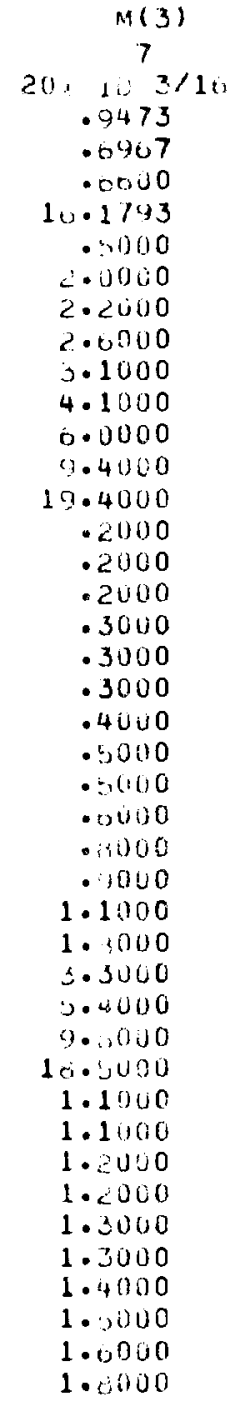

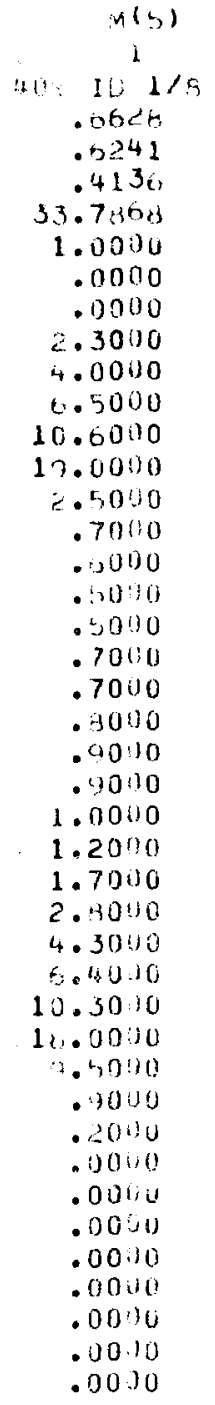

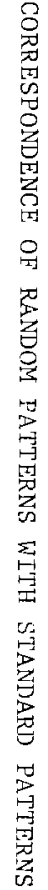

\begin{tabular}{|c|c|}
\hline $4(4)$ & $M(b)$ \\
\hline 21 & I \\
\hline $16 \%$ I) $1 / 16$ & Ho: If $1 / 5$ \\
\hline .7441 & $.062 \theta$ \\
\hline .5460 & .0241 \\
\hline .4137 & $.413 i$ \\
\hline 37.9257 & 33.7360 \\
\hline 2.0000 & 1.0000 \\
\hline .0000 & .0000 \\
\hline .0000 & .0000 \\
\hline ن ن 000. & 2.3000 \\
\hline .0000 & 4.0000 \\
\hline .0000 & 0.5000 \\
\hline 3.8000 & 10.6000 \\
\hline 0.2000 & 13.00130 \\
\hline 9.3400 & 2.5000 \\
\hline 1.0000 & .7000 \\
\hline $.1000 \mathrm{j}$ & \\
\hline .1000 & .3000 \\
\hline .1000 & . \\
\hline . $10 \cap 0$ & $.700: 0$ \\
\hline .1000 & .7000 \\
\hline .1000 & .3000 \\
\hline .1000 & .9010 \\
\hline .1000 & . $90 \div 0$ \\
\hline .1000 & 1.0000 \\
\hline .2000 & 1.2000 \\
\hline 1.0000 & 1.7000 \\
\hline 1.7000 & 2.8000 \\
\hline 3.0000 & 4.3000 \\
\hline 5.0000 & $5.40 .10 \mathrm{j}$ \\
\hline 7.7000 & $10.30: 10$ \\
\hline $10 \cdot \operatorname{conn}$ & 11.0000 \\
\hline $.00 n 0$ & $24,0 ! 10$ \\
\hline .0500 & .9000 \\
\hline .00000 & .2010 \\
\hline .00000 & .0000 \\
\hline .0000 & . 00 lia \\
\hline .0000 & נ10000 \\
\hline . 0000 & .0010 \\
\hline .0000 & .0060 \\
\hline .0000 & .0030 \\
\hline .0000 & .00 .10 \\
\hline . & .0030 \\
\hline
\end{tabular}




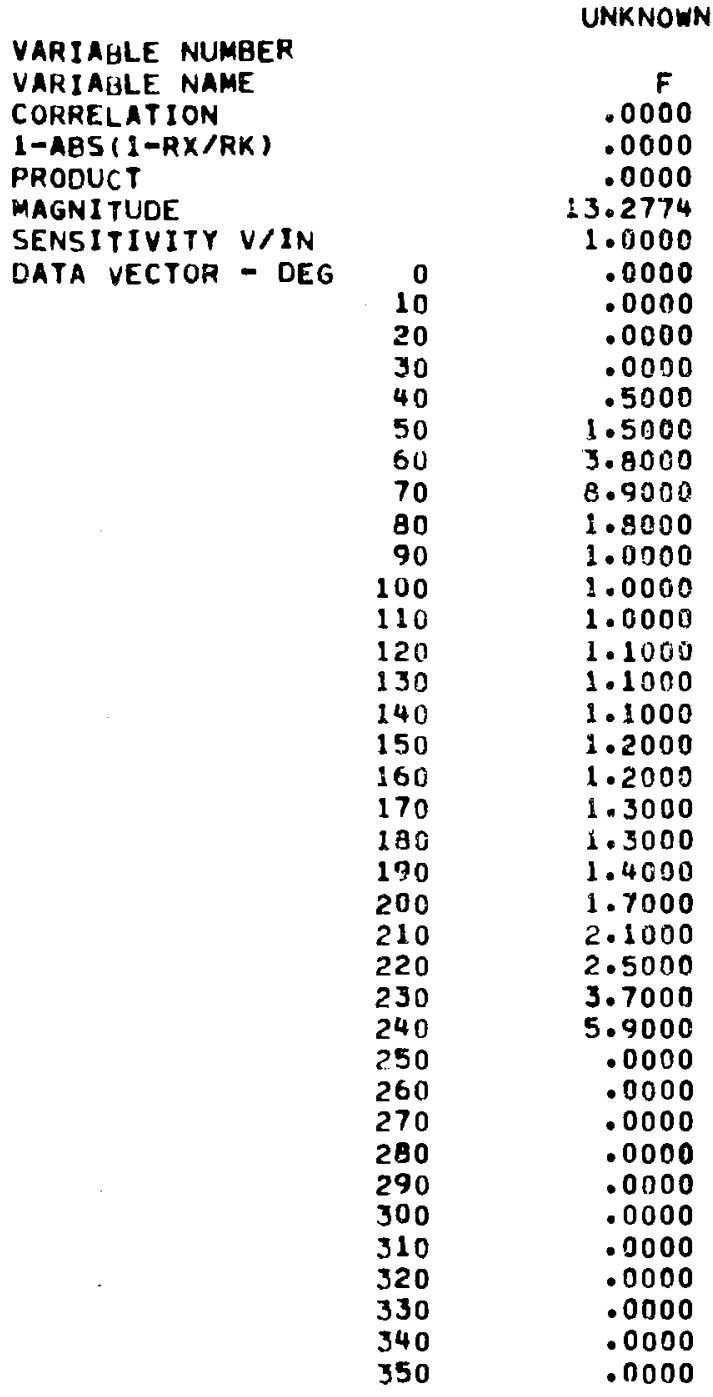

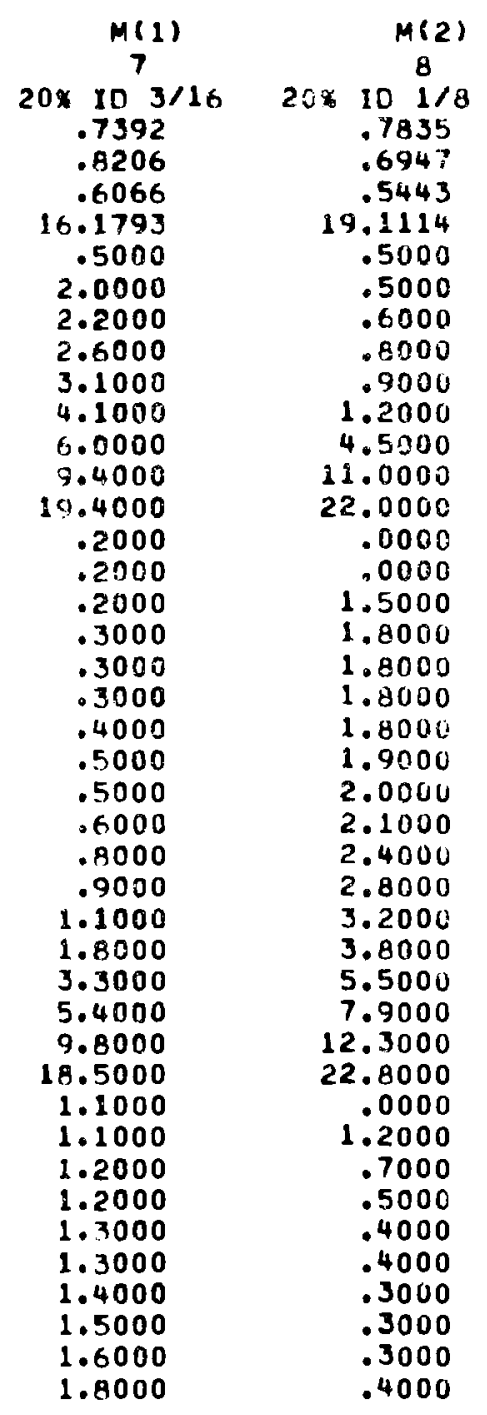

$14(3)$
9
$40 \times 101116$
.7225
.7392
.5341
10.5310
.5000
.1000
.1000
.1000
.1000
.2000
.2000
.2000
10.0000
10.0000
4.0000
3.0000
2.5000
2.1000
1.9000
1.8000
1.7000
1.7000
1.8000
1.9000
2.0000
2.1000
2.4000
3.0000
4.0000
6.0000
10.0000
3.5000
.9000
.1000
.1000
.1000
.1000
.1000
.1000
.1000
.1000

$14(4)$
2
101116
.7501
.5654
.4241
23.4840
1.0000
.0000
.0000
.1000
1.2000
2.9000
5.0000
8.5000
13.3000
.0000
.0000
.0000
.0000
.0000
.0000
.0000
.0000
.0000
.0000
.0000
.1000
.1000
1.1000
2.2000
4.8000
8.2000
13.0000
1.3000
.6000
.2000
.1000
.2000
.1000
.0000
.0000
.0000
.0000

$M(5)$
21
$80 \% 101116$
.9280
.3501
.3249
37.9257
2.0000
.0000
.0000
.0000
.0000
.0000
3.8000
6.2000
9.8000
1.0000
.1000
.1000
.1000
.1000
.1000
.1000
.1000
.1000
.1000
.2000
1.0000
1.7000
3.0000
5.0000
7.7000
10.6000
.0000
.0000
.0000
.0000
.0000
.0000
.0000
.0000
.0000
.0000
.0000


VARIALLE NUMBER

VARIAUL NAME

CORRE LAT IOIN

$1-\operatorname{ARS}(1-R x / R K)$

PROTUET

MAGNI TUDE

SENSI I IVITY $V / I N$

DATA VECTOR - DEC
UNK:OWN

$M(1)$
11
3116
.0780
.9599
.9380
531.0273
10.0000
3.7000
5.2000
7.9000
11.8000
17.0000
24.1000
70.4000
1.9000
.8000
.6000
.1000
.1000
.1000
.7000
.9000
1.1000
1.6000
2.2000
3.2000
4.0000
7.0000
10.4000
15.9000
24.5000
15.0000
.0000
.0000
.0000
.0000
.0000
.0000
.0000
.0000
1.0000
1.3000
2.2000

\begin{tabular}{|c|c|}
\hline$N(c)$ & $M(3)$ \\
\hline 15 & 4 \\
\hline $100=110$ & $20 \times 0,3 / 16$ \\
\hline.$<521$ & $-65 \therefore 260$ \\
\hline .2440 & -1.1229 \\
\hline 291.6310 & 7.5000 \\
\hline 10.0000 & .2000 \\
\hline 2.4000 & .0000 \\
\hline 3.4000 & .0000 \\
\hline 4.9000 & .0000 \\
\hline 6.9000 & .0000 \\
\hline 9.8000 & .0000 \\
\hline 13.2000 & .0000 \\
\hline .0000 & .0000 \\
\hline .0000 & • 0.000 \\
\hline .0000 & .0000 \\
\hline .0000 & 10.0000 \\
\hline .0000 & 9.4000 \\
\hline .0000 & 5.0000 \\
\hline .0000 & 16.0000 \\
\hline .0000 & 10.0000 \\
\hline .0000 & $11 \cdot 8000$ \\
\hline .0000 & .0000 \\
\hline .0000 & .0000 \\
\hline 1.1000 & .0000 \\
\hline 1.9000 & .0000 \\
\hline 3.0000 & .0000 \\
\hline 4.5000 & .0000 \\
\hline 6.4000 & .0000 \\
\hline 9.9000 & .0000 \\
\hline 13.65000 & .0000 \\
\hline 11.0000 & .0000 \\
\hline 1.0000 & $4 . y 300$ \\
\hline .6000 & $1 \cup \cdot 0000$ \\
\hline .4000 & $7 \cdot 2000$ \\
\hline .2000 & 8.7000 \\
\hline .2000 & 10.0000 \\
\hline .2000 & $11 . ? 000$ \\
\hline .1000 & 12.9000 \\
\hline .1000 & $7 \cdot 3000$ \\
\hline .1000 & $.00 \cup 0$ \\
\hline 1.0000 & .0000 \\
\hline & \\
\hline
\end{tabular}

$4(4)$
13
401.3116
.1093
-25.9708
-2.8389
18.2241
.2000
.8000
.9000
1.1000
1.3000
2.2000
4.2000
7.9000
10.0000
11.9000
13.8000
15.7000
18.8000
22.2000
26.9000
32.6000
40.8000
19.5000
.0000
.0000
.0000
.0000
.0000
.0000
.0000
.0000
.0000
.0000
.0000
.0000
.0000
8.8000
14.2000
19.9000
27.3000
37.0900
.9000

$M(5)$

12 4 ) I $1 /$ $-3.01321$ $-3.0662$ 15.8639 .2000 - 90110 - 9000 .1000 .1000 .1000 .1000 .2000 .2000 .2000 .3000 . 30 ن 0 .0000 1.0000 7.3000 14.9000 24.1000 34.1000 .0000 .00110 .0040 $.001: 0$ .0000 .0000 .0000 .0000 .0000 6.5000 $\because .3010$ 12.00110 15.3000 19.4000 4.000 29.1000 $2 \% .7000$ 
VARIABLE NUMBER

VARIAHLE NAME

CORRELATION

1-ABS $(1-R X / R K)$

PRODUCT

MAGNI TUDE

SENSITIVITY V/IN

DATA VECTOR - DEG
UNKNOWN

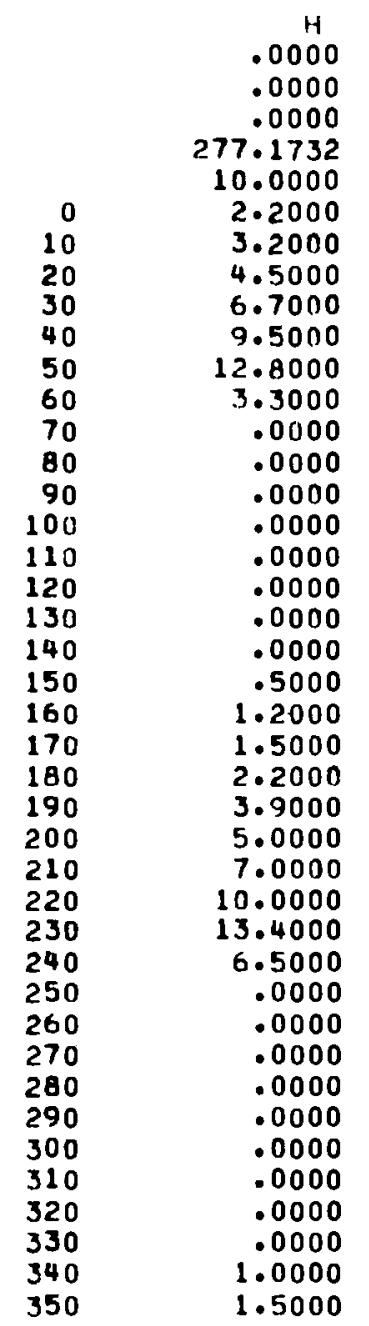

$M(1)$

$100 \times 1 / 8$
.9775

.9504

.9291

291.6316

10.0000
2.4000

2.4000
3.4000

4.9000

6.9000

9.8000

13.2000

.0000

.0000

.0000

.0000

.0000

.0000

.0000

.0000

.0000

.0000

.0000
1.1000

1.1000
1.9000

1.9000
3.0000

3.0000
4.5000

6.4000

9.9000

13.8000

11.0000

1.6000

.6000

.4000

.2000

.2000

.2000

.1000

.1000

.1000

1.0000
1.6000

$M 121$
11
$100 \% 3116$
.9551
.5220
.4985
531.0273
10.0000
3.7000
5.2000
7.9000
11.8000
17.0000
24.1000
20.4000
1.9000
.8000
.6000
.1000
.1000
.1000
.7000
.9000
1.1000
1.6000
2.2000
3.2000
4.9000
7.0000
10.4000
15.9000
24.5000
15.0000
.0000
.0000
.0000
.0000
.0000
.0000
.0000
.0000
1.0000
1.3000
2.2000

$M(3)$
4
$208003 / 16$
.0000
-34.6030
$=.0000$
7.5600
.2000
.0000
.0000
.0000
.0000
.0000
.0000
.0000
.0000
.0000
10.0000
9.4000
9.6000
10.0000
10.8000
11.9000
.0000
.0000
.0000
.0000
.0000
.0000
.0000
.0000
.0000
.0000
9.9000
10.9000
7.2000
8.7000
10.0000
11.2000
12.9000
7.5000
.0000
.0000
.0000

$M(4)$
19
$1003 / 16$
.9441
-1.0550
-.9960
90.7290
2.0000
2.0000
3.2000
5.1000
8.9000
14.9000
27.0000
.0000
.0000
.0000
.0000
.0000
.0000
.0000
.0000
.0000
.0000
.5000
1.0000
1.8000
3.7000
5.3000
8.6000
14.1000
25.8000
1.0000
.0000
.0000
.0000
.0000
.0000
.0000
.0000
.0000
.3000
.7000
1.1000

(4)

$M(5)$
20
110118
.8887
-1.1212
-.9964
88.8041
2.0000
.0000
2.0000
4.0000
6.9000
11.0000
18.4000
20.5000
.0000
.0000
.0000
.0000
.0000
.0000
.0000
.0000
.0000
.5000
1.0000
1.9000
4.0000
6.8000
10.1000
17.4000
23.5000
.0000
.0000
.0000
.0000
.0000
.0000
.0000
.0000
.0000
.0000
.0000
.0000


VARIABJLE NUMEER VAFIAISLE NAME CORRELATION

$1-A[S(1-R \times / R K)$

PRODUCT

MAGNI TUDE

SENSITIVITY VIIN

LATA VECTOR - DEG
URNKNONIN

1
.0000
.0000
.0000
85.2351
1.0000
.0000
.0000
.0000
12.0000

12.0000

21.8000

37.3000

34.6000

2.0000

.0000

.0000

. j000

- 0000

.0000

- 0000

.0000

.0000

1.0000

3.5000

3.5000
8.0000

13.5000

23.7000

38.3000

42.0000

1.0000

.5000

.0000

.0000

.0000

.0000

.0000

.0000

.0000

.0000

.0000
M(1)

80* 20

.8554

.9598

.8210

83.8041

.0000

2.0000

4. 1000

.9000

11.0000

18.4000

20.5000

.0000

.0000

.0000

.0000

.0000
.0000

.0000

.0000

.0000

1.0000

1.9000
4.0000

4.0000
6.8 .000

10.1000

17.4000

23.5000

.0000

0000

.0000
.0000

.0000
.0000

.0000

.0000

.0000

.0000

.0000

.0000

.0000
19

BOx IU $3 / 10$

.7697

.7231

90.7290

2.0000

2.0000

3.2000

5.1000

8.9000
14.9000

27.0000

.0000

000

.0000

.0000

.0000
.0000

.0000

.0000

.0000

.0000

.5000

1. 0000

1.8000

3.7000

5. 3000

8.6000

14.1000

25.18000

1.000u

.000

.0000

.0001

.0000

.0000

.0000

nnos

.3000

1.1000
100.14

.0647
.9655

.9855
.0748

80.4834

2.0000

5.4000

7.2000
4.8000

12.9000

17.0000

19.9000

.5000

.5000

.6000

.7000

-9u00

1.1000

1.2000

1.5000

2.0000

2.7000

4.5000

0.1000

8.2000

$11 \cdot 0000$

15.5000

- 0000

.0000

- luvo

- uojoo

-1000
1.1000

1. 3000

1.5000

1. $: 000$

2.0000

3.2000
4.2000
$M(4)$

100 OD 2 ? 04 H

.7176

.6128
.4397

61.4429

1.0000

5.0000

7.9000

11.3000

16.4000
22.2000

28.0000

1.1000

1.2000

1.3000

1.0000
1.9000

¿. 0000

2. 1000

2.6000

3.0000

3.8000

4.3000

5.5000

7.2000

9.7000
12.4000

17.1000

23.0000

28.1000

.0000

.0000

$.00 n 0$
.0000

. 0000

- 0000

.0000

- 0000

.0000

.0000

2.4000

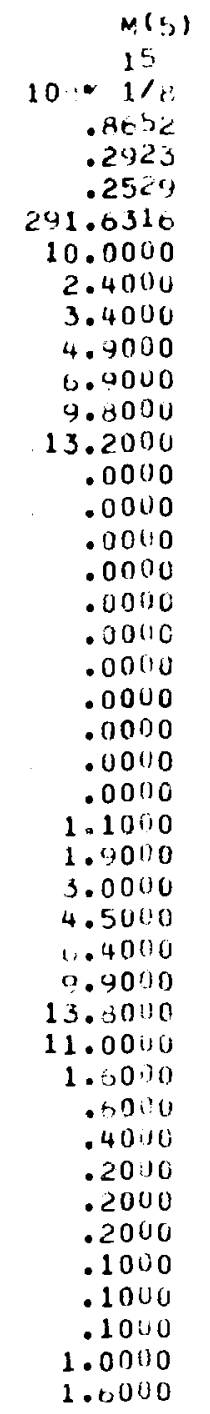




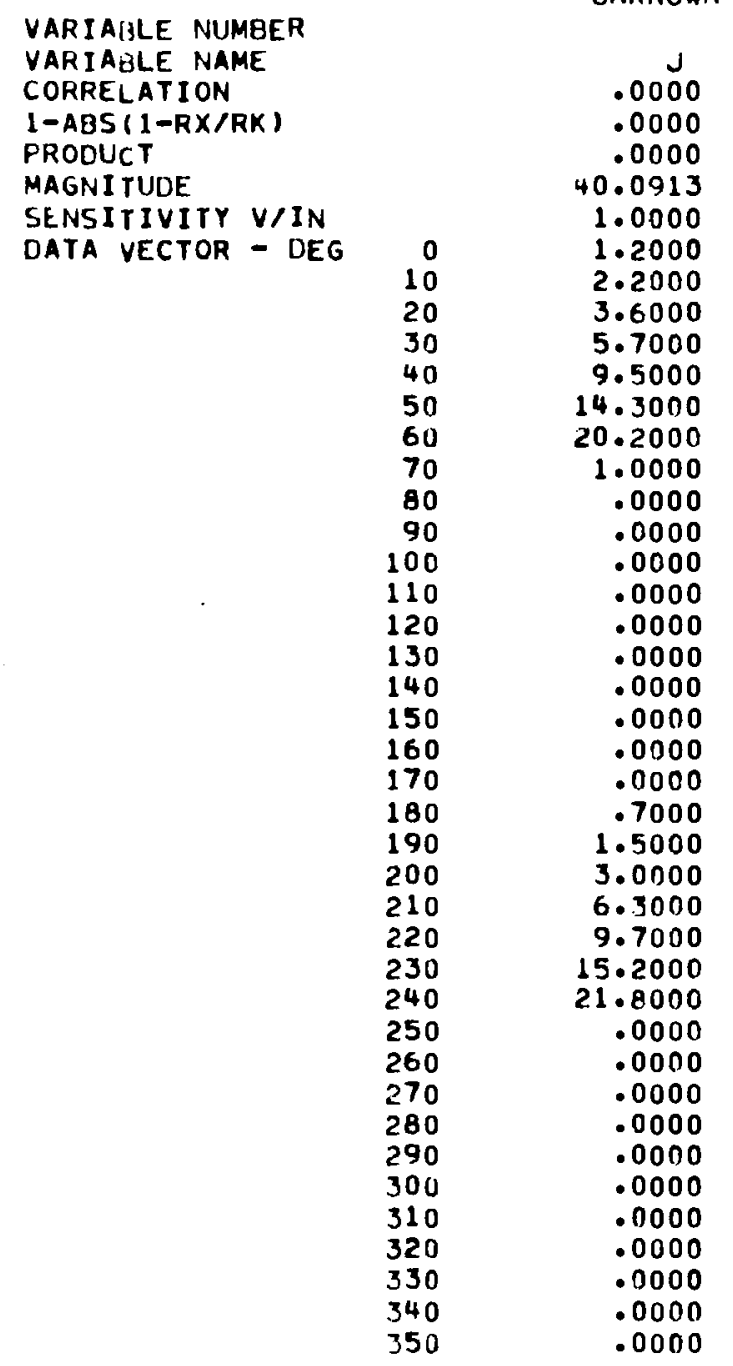

$M(1)$
3
$407.3 / 10$
.9884
.8753
.8651
35.6448
1.0000
1.1000
2.0000
2.9000
4.6000
6.8000
11.2000
20.7000
.0000
.0000
.0000
.0000
.0000
.0000
.0000
.0000
.0000
.0000
.0000
.0000
1.5000
2.5000
4.0000
6.7000
11.0000
21.1000
.0000
.0000
.0000
.0000
.0000
.0000
.0000
.0000
.0000
.0000
.0000

$M(2)$
1
$40 \times 101 / 8$
.9454
.8134
.7690
33.7808
1.0000
.0000
.0000
2.3000
4.0000
6.5000
10.6000
19.0000
2.5000
.7000
.6000
.5000
.5000
.7000
.7000
.8000
.9000
.9000
1.0000
1.2000
1.7000
2.8000
4.3000
6.4000
10.3000
18.0000
9.5000
.9000
.2000
.0000
.0000
.0000
.0000
.0000
.0000
.0000
.0000

$M(3)$
21
$804101 / 16$
.8045
.9429
.7586
37.9257
2.0000
.0000
.0000
.0000
.0000
.0000
3.1000
0.2000
90000
1.0000
.1000
.1000
.1000
.1000
.1000
.1000
.1000
.1000
.1000
.2000
1.0000
1.7000
3.0000
5.0000
7.7000
10.0000
.0000
.0000
.0000
.0000
.0000
.0000
.0000
.0000
.0000
.0000
.0000

$M(4)$
100
$200.040 R$
.6524
.6525
.4257
01.4429
1.0000
5.0000
7.9000
11.3000
16.4000
22.2000
28.0000
1.1000
1.2000
1.3000
1.6000
1.9000
2.0000
2.1000
2.6000
3.0000
3.8000
4.3000
5.5000
7.2000
9.7000
12.4000
17.1000
23.0000
28.1000
.0000
.0000
.0000
.0000
.0000
.0000
.0000
.0000
.0000
.0000
.0000
2.4000
$M(5)$
20
$101 / 8$
.8186 .4515 .3696
80.8041 2.0000
.0000 .0000 2.0000
4.0000 4.0000
6.90000 11.0000 18.4000 20.5000 .0000 .0000 .0000 .0000 .0000 .0000 .0000 .0000 $.001 j 0$ .5000 1.0000 1.0000 1.9000 4.0000 6.8000 10.1000 17.4000 .0000 .0000 .0000 .000 .0000 .0000 .0000 .00130 .0000 .0000 .0030 opría .0000


VARIAULE NIJMBER VAKIALILE NAME CORRELATION

$1-A B S(1-R X / R K)$

PRODUCT

MAGNI TUOE

SENSITIVITY $V / I N$

DATA VECTOR - DEG
UNKHOWN

$k$
.0000
.0000
.0000
56.8086
1.0000
8.9000
10.8000
14.0000
17.8000
23.2000
.0000
.0000
.0000
.0000
.0000
.0000
.0000
.0000
.0000
.0000
.5000
1.0000
2.3000
4.2000
7.3000
11.2000
15.7000
22.6000
29.3000
4.0000
2.3000
1.8000
1.5000
1.7000
2.0000
2.7000
3.2000
3.6000
4.5000
5.8000
7.1000

19

$M(1)$

22

$1000 \Gamma .0408$

.8535

.9256
.7900

61.4429

1.0000

5.0000

11.3000

16.4000

22.2000

28.0000
1.1000

1.2000

1.3000

1.6000

1.9000
2.0000

2.0000
2.1000

2.6000

3.0000

3.8000

4.3000

5.5000

7.2000

9.7000

12.4000

17.1000

23.0000

20.1000

.0000

.0000

$-9000$

.0000
.0000

.0000

.0000

.0000

.0000

.000

.0000

2.4000
$M(2)$

$100 \times 1 / 16$

.8711

.6575

.5727

86.4884
2.0000

5.4000

7.2000

12.9000

12.9000

19.0000

.5000

.5000

.6000

.7000

.8000
.9000

1.1000

1.2000

1.5000

2.0000

2.7000

3.3000

4.5000
6.1000

8.2000

11.6000

15.9000

16.0000

.0000

.0006

$.000 u$

.5000

1.1000

1.3000

1.9000

1.9000

2.6000

4.2000
$N(3)$

19

30* $1: 3 / 1$.

- 7564

.6268
.4741

90.7290

2.0000

2.0000

3.2000
5.1000

त. 9000

14.9000

27.0000

.0000

.0000

.0000

- 5000

.0000

.0000

.0000

.0000

.5000

1.0000

1. 3000

S. 7000

1.0000

14.1000

25.6000

1.0000

.0000

.0000

.0000
.0000

.4000

.0000

.0000

.0000

.3000

$\cdot 7000$
1.1000

$1414)$
20
$10.1 / 8$
.7170
.6404
.4596
88.8041
2.0000
.0000
2.0000
4.0000
6.9000
11.0000
18.4000
20.5000
.0000
.0000
.0000
.0000
.0000
.0000
.0000
.0000
.0000
.5000
1.0000
1.9000
4.0000
6.8000
10.1000
17.4000
23.5001
.0000
.0000
.0000
.0000
.0000
.0000
.0000
.0000
.0000
.0000
.0000
.0000

20

$4(5)$

21

(8) 1 : $1 / 10$

$.422^{32}$

.5015
.2115

37.9257

2.0000

.0000

.0040

.0000

.0000

.0000
.0000

3.8000

6.2000

9.8000

1.0000

.1000

.1000

.1000

.1000
.1000

.1000
$.10 u 0$

.1040

.1000

$.10 \cup 0$

.1000

- 1000

1. 0000

1. 70110

3.0000

$5.00 \cup 0$

7.7000

$10.6,000$

.0000

.0000

.00110

.0000

.0000

.0000

.0000

.0000

. v0u0

.0000

.0000 


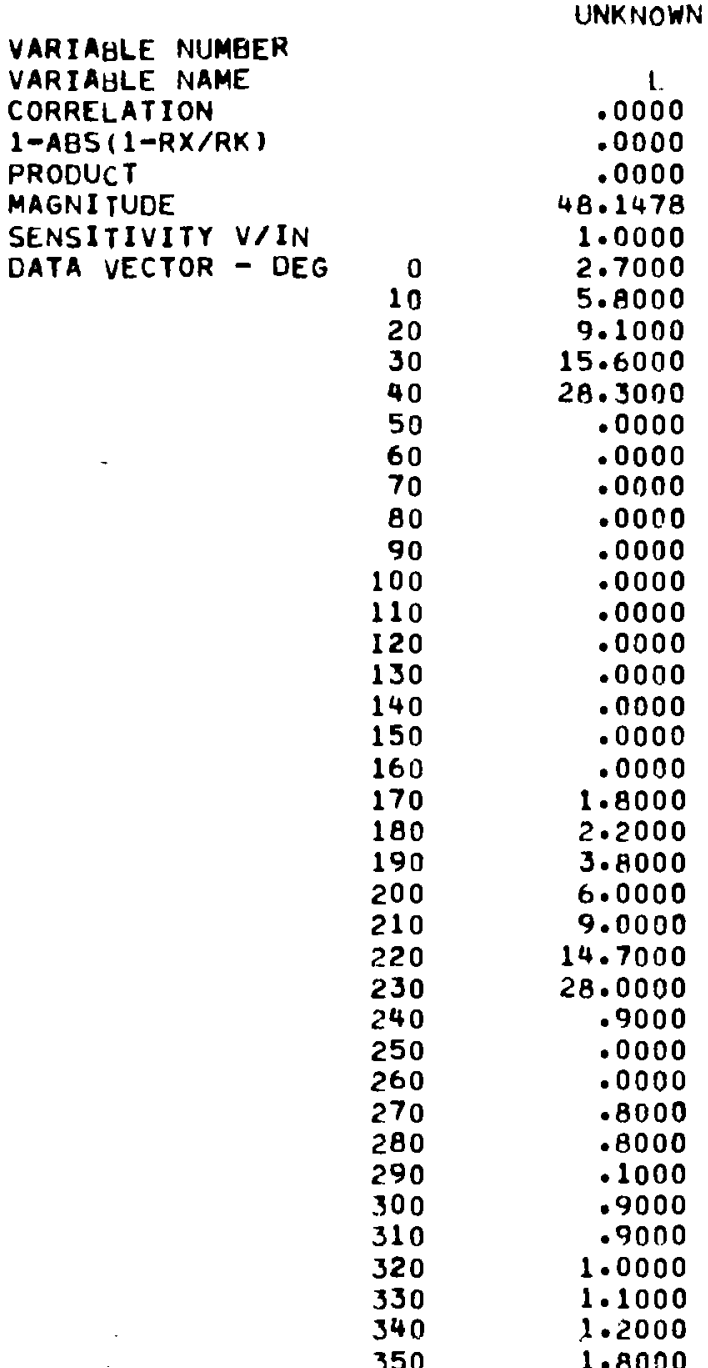

$M(1)$
22
$10000.040 R$
.8337
.7836
.6533
61.4429
1.0000
5.0000
7.9000
11.3000
16.4000
22.2000
28.0000
1.1000
1.2000
1.3000
1.6000
1.9000
2.0000
2.1000
2.6000
3.0000
3.8000
4.3000
5.5000
7.2000
9.7000
12.4000
17.1000
23.0000
28.1000
.0000
.0000
.0000
.0000
.0000
.0000
.0000
.0000
.0000
.0000
.0000
2.4000

$M(2)$
14
$100 \times 1116$
.8268
.5567
.4603
86.4884
2.0000
5.4000
7.2000
9.8000
12.9000
17.0000
19.9000
.5000
.5000
.6000
.7000
.8000
.9000
1.1000
1.2000
1.5000
2.0000
2.7000
3.3000
4.5000
6.1000
8.2000
11.6000
15.9000
16.0000
.0000
.0000
.0000
.0000
.5000
1.1000
1.3000
1.5000
1.9000
2.6000
3.2000
4.2000

$M 131$
19
$80 \times 103 / 16$
.7754
.5307
.4115
911.7290
2.0000
2.0000
3.2000
5.1000
8.9000
14.9000
27.0000
.0000
.0000
.0000
.0000
.0000
.0000
.0000
.0000
.0000
.0000
.5000
1.0000
1.8000
3.7000
5.3000
8.6000
14.1000
25.8000
1.0000
.0000
.0000
.0000
.0000
.0000
.0000
.0000
.0000
.3000
.7000
1.1000

$M(4)$
20
$807101 / 8$
.7174
.5422
.3889
88.8041
2.0000
.0000
2.0000
4.0000
6.9000
11.0000
18.4000
20.5000
.0000
.0000
.0000
.0000
.0000
.0000
.0000
.0000
.0000
.5000
1.0000
1.9000
4.0000
6.8000
10.1000
17.4000
23.5000
.0000
.0000
.0000
.0000
.0000
.0000
.0000
.0000
.0000
.0000
.0000
.0000

$M(5)$
3
$103 / 16$
.4588
.6492
.2978
35.6448
1.0000
1.1000
2.0000
2.9000
4.6000
6.8000
11.2000
20.7000
.0000
.0000
.0000
.0000
.0000
.0000
.0000
.0000
.0000
.0000
.0000
.0000
1.5000
2.5000
4.0000
6.7000
11.0000
21.1000
.0000
.0000
.0000
.0000
.0000
.0000
.0000
.0000
.0000
.0000
.0000


VARIAULE NUMBER

VARIAULE MAME

COKRELATION

$1-A B S(1-R X / R K)$

HFODUCT

MAGNI TUDE

SETSITIVITY V/IN

DATA VECTOR - DEG
UNKIIOWN

\begin{tabular}{|c|c|}
\hline in & 100 in $.040 \mathrm{D}$ \\
\hline .0000 & $.24+39$ \\
\hline .0000 & .7021 \\
\hline .0000 & .1755 \\
\hline 43.1335 & 61.4429 \\
\hline 1.0000 & 1.0000 \\
\hline .0000 & 5.0000 \\
\hline .0000 & 7.9000 \\
\hline .0000 & $11 . \times 000$ \\
\hline .0000 & 16.4000 \\
\hline $.00 \cap 0$ & 22.2000 \\
\hline .0000 & 28.0000 \\
\hline .5000 & 1.1000 \\
\hline 1.0000 & 1.2000 \\
\hline 1.3000 & 1.3000 \\
\hline .9000 & 1.6000 \\
\hline 1.6000 & 1.9000 \\
\hline 4.3000 & 2.0000 \\
\hline 5.8000 & 2.1000 \\
\hline 14.7000 & 2.6000 \\
\hline 24.0000 & 3.0000 \\
\hline 14.4000 & 3.8000 \\
\hline 9.1000 & 4.3000 \\
\hline 7.0000 & 5.5000 \\
\hline 5.5000 & 7.2000 \\
\hline 4.6000 & 9.7000 \\
\hline 4.1000 & 12.4000 \\
\hline 3.8000 & 17.1000 \\
\hline 3.7000 & 2.3 .0000 \\
\hline 3.7000 & 28.1000 \\
\hline 3.8000 & .0000 \\
\hline 4.0000 & .0000 \\
\hline $4 \cdot 2000$ & .0000 \\
\hline 4.7000 & .0000 \\
\hline 5.6000 & .0000 \\
\hline 7.3000 & .0000 \\
\hline 10.4000 & .0000 \\
\hline 16.9000 & .0000 \\
\hline .0000 & $\cdot 0 n 00$ \\
\hline .0000 & .0000 \\
\hline .0000 & .0000 \\
\hline .0000 & 2.4000 \\
\hline
\end{tabular}

4ux $\operatorname{In} 1 / \theta$

.2017

.7232
.1454

.1459
33.7868
1.0000

1.0000

.0000

2.3000

4.0000

6.5000

10.6000

19.0000

2.5000

.7000

.6000

.5000
.5000

. .5000

.7000

.8000

.9000

.9040

1.0000
1.2000

1.2000
1.7000

1.7000
2.8000

4.3000

0.4000

10.3000

18.0000

0.5000

.3000
.9000

.9000
.2000

.2000
.0000

.0000

.0000

.0000

.0000

.0000

.0000

.0001
$M(3)$

21

804 ID $1 / 1$ :

- 1636

.8626

.1411
37.4257

2.0000

.0000

.0000

.0000

.0000

.0000

6.2000

9.83000

1.0000

.1000

.1000

$-1000$

- 1000

.1000

.1000

.1000

.1000

.2000

1.0000

1.7060

3.0000

7.7000

$10 \cdot 6000$

.0000

- juilo

- 0000

- ju00

.0000

.0000

.0000

.0000

. .0000

$-000$

.0000
.0000
$.98(4)$

$100 \times 1 / 16$

.2575

.4988
.1185

86.4884
2.0000

5.4000

7.2000

$9.80 \cap 0$

12.9000

17.0000

19.9000

.5000
.5000

.0000

.7000

- 807000

.9000

1.1000

1.2000

1.5000

2.0000

2.7000

4.5000

6.1000

8.2000

11.6000

16.0090

.0000

.0009

- 0 i)

.0000
.5000

.1000

1.3000

1.5000

1.9000

3.2000

3.2000
4.2000

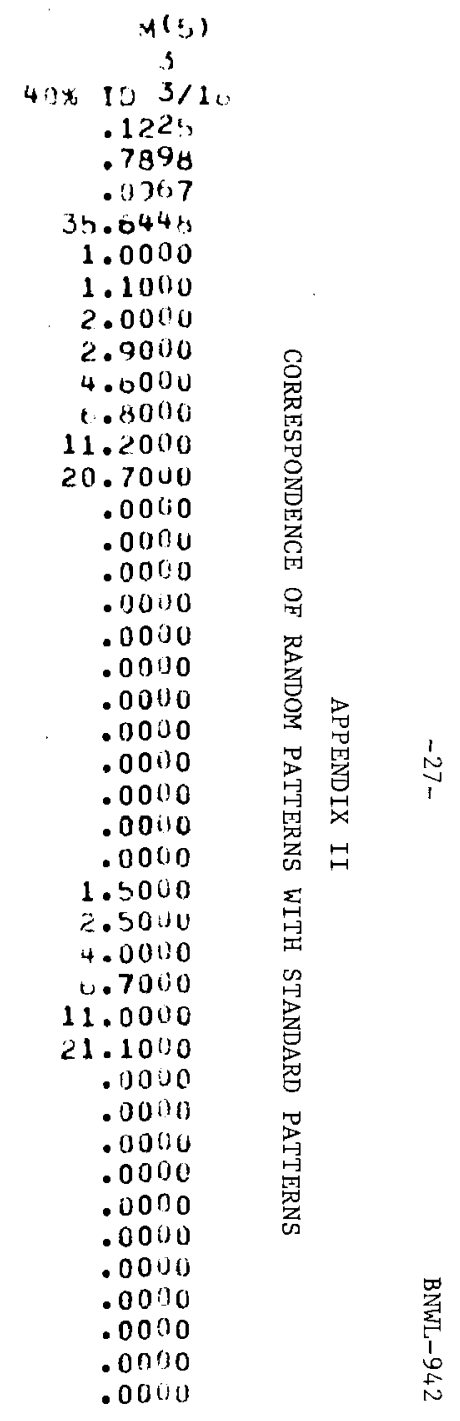




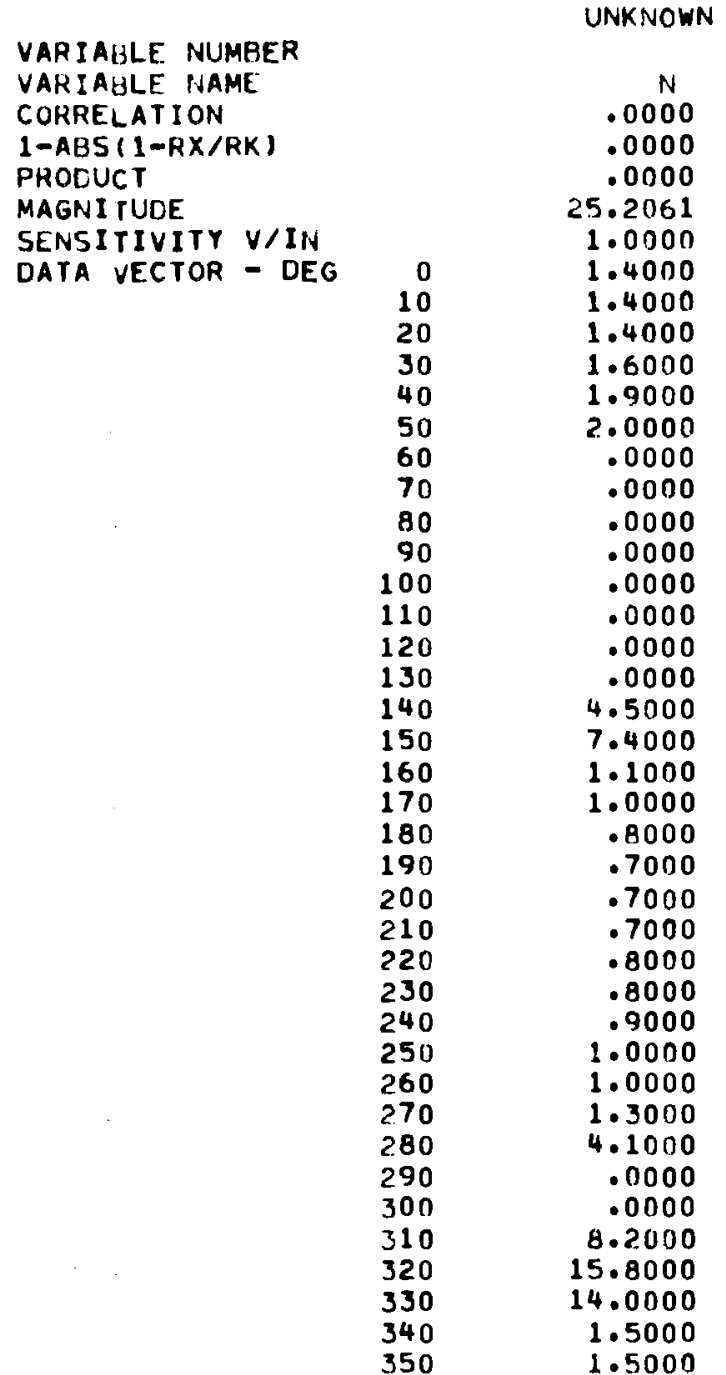

$M(1)$
13
$407003 / 16$
.5921
.6169
.3652
18.2241
.2000
.8000
.9000
1.1000
1.3000
2.2000
4.2000
7.9000
10.0000
11.9000
13.8000
15.7000
18.8000
22.2000
26.9000
32.6000
40.8000
19.5000
.0000
.0000
.0000
.0000
.0000
.0000
.0000
.0000
.0000
.0000
.0000
.0000
.0000
8.8000
14.2000
19.9000
27.3000
37.9000
.9000

$M(2)$
12
$40 \% 101 / 8$
.6406
.4111
.2658
15.8639
.2000
.9000
.9000
.1000
.1000
.1000
.1000
.2000
.2000
.2000
.3000
.3000
.6000
1.0000
2.0000
7.3000
14.9000
24.1000
34.1000
.0000
.0000
.0000
.0000
.0000
.0000
.0000
.0000
.0000
6.5000
9.3000
12.0000
15.3000
19.4000
24.0000
29.1000
33.7000
28.7000

$M(3)$
17
$50 \% .0040 R$
.3529
.3087
.1089
14.0036
.3000
14.3000
1.0000
.2000
.0000
.0000
.0000
.0000
.0000
.0000
.0000
.0000
.0000
.0000
.0000
.0000
.0000
7.0000
10.0000
13.7000
1.0000
.0000
.0000
.0000
.0000
.0000
.0000
.0000
.0000
.0000
.0000
.0000
.0000
5.0000
7.2000
9.9000
12.4000

$M(4)$
1
$4091 / 8$
.1207
.7460
.0900
33.7808
1.0000
.0000
.0000
2.3000
4.0000
6.5000
10.6000
19.0000
2.5000
.7000
.6000
.5000
.5000
.7000
.7000
.8000
.9000
.9000
1.0000
1.2000
1.7000
2.8000
4.3000
6.4000
10.3000
18.0000
9.5000
.9000
.2000
.0000
.0000
.0000
.0000
.0000
.0000
.0000
.0000

$M(5)$
8
$20 \% 101 / 8$
.1172
.6811
.0795
19.1114
.5000
.5000
.6000
.8000
.9000
1.2000
4.5000
11.0000
22.0000
.0000
.0000
1.5000
1.8000
1.8000
1.8000
1.8000
1.9000
2.0000
2.1000
2.4000
2.8000
3.2000
3.8000
5.5000
7.9000
12.3000
22.8000
.0000
1.2000
.7000
.5000
.4000
.4000
.3000
.3000
.3000
.4000

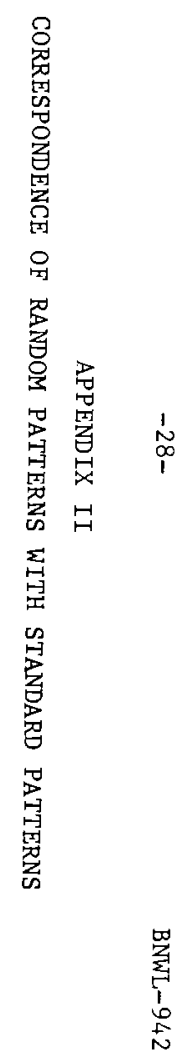


VARI AGLE NUMBER

VARIABLE NAME

1-ABS $(1-R X / R K)$

PRODUCT

MAGNI TUDE

SENSITIVITY $V / I N$

DATA VECTOR - DEG
UNKNOWN

0
.0000
.0000
.0000
37.0800
1.0000
.0000
.0000
.0000
.0000
.0000
.0000
.0000
.0000
.0000
10.7000
12.6000
12.1000
13.4000
14.5000
13.2000
3.3000
3.0000
2.9000
2.7000
2.8000
2.8000
2.8000
2.9000
3.0000
3.1000
3.3000
3.9000
5.0000
4.3000
5.1000
5.8000
7.2000
7.3000
7.3000
.0000
.0000

$40 \%$ ID . 1 A10

0.1634

.1634
1.7868

33.7868
1.0000

1.0000
.0000

.0000

2.3000

4.0000

6.5000

10.6000

19.0000

2.5000

.7000

.6000

.5000

.5000

.7000
.7000

.8000

.9000

.9000

1.0000

1.2000

1.7000

2. 8.000

2.8 .000
4.3000

6.4000

10.3000

18.0000

9.5000

.9000

.2000

.0000
.0000

.0000

.0000

.0000

.0000

. 0000
$M(2)$

BOX ID $1 / 10$

.1360

.1329
37.9257

37.9257
2.0000

2.0000
.0000

.0000

.0000

.0000

.0000

3.8000

6.2000

9.8000

1.0000

.1000

.1000

11000
.1000

.1000

.1000

.1000

.1000

.2000

1.0000

1.7000
3.0000

3.0000
5.0000

5.0000
7.7000

10.6000

.0000

.0000

.0000

.0000

.0000
.0000

.0000

.0000

.0000

.0000

.0000
$M(3)$

22

$100 \mathrm{OQ} \cdot 040 \mathrm{R}$

.2167

.1308

61.4429

1.0000

5. 0000

7.9000
11.3000

11.3000

22.2000

28.0000

1.1000

1.2000
1.3000

1.6000

1.4000

2.0000

2.1000

2.6000

j. 0000

3.8000

4.3000

5.5000

7.2000

9.7000

12.4000

17.1000

23.0000

28.1000

.0000

.0000

.0000

.0000

.0000

.0000

.0000

.0000

.0000

.0000

2.4000
$4(4)$
3

40* I0 $3 / 16$

- 1061

.1018

35.6448

1.0000

1.1000

2.0000

4.6000

6.8000

11.2000

20.7000

.0000

.0000

.0000

.0000

.0000

.0000

.0000

.0000

.0000

1.5000

2.5000

6.7000
11.0000

21.1000

.0000

.0000

.0000

.0000

.0000

.0000

.0000

.0000

.0000

$1.15)$
14
100.111
.213
.4287
.0917
86.4884
2.0000
5.4000
7.2000
9.8000
12.9000
17.0000
19.9000
.5000
.5000
.0000
.7000
.8000
.9000
1.1000
1.2000
1.5000
2.0000
2.7000
3.3000
4.5000
1.1000
8.2000
11.6000
15.9000
10.0000
.0000
.0000
.0000
.0000
.50110
1.1000
1.3000
1.5000
1.9000
2.6000
3.2000
4.2000
4




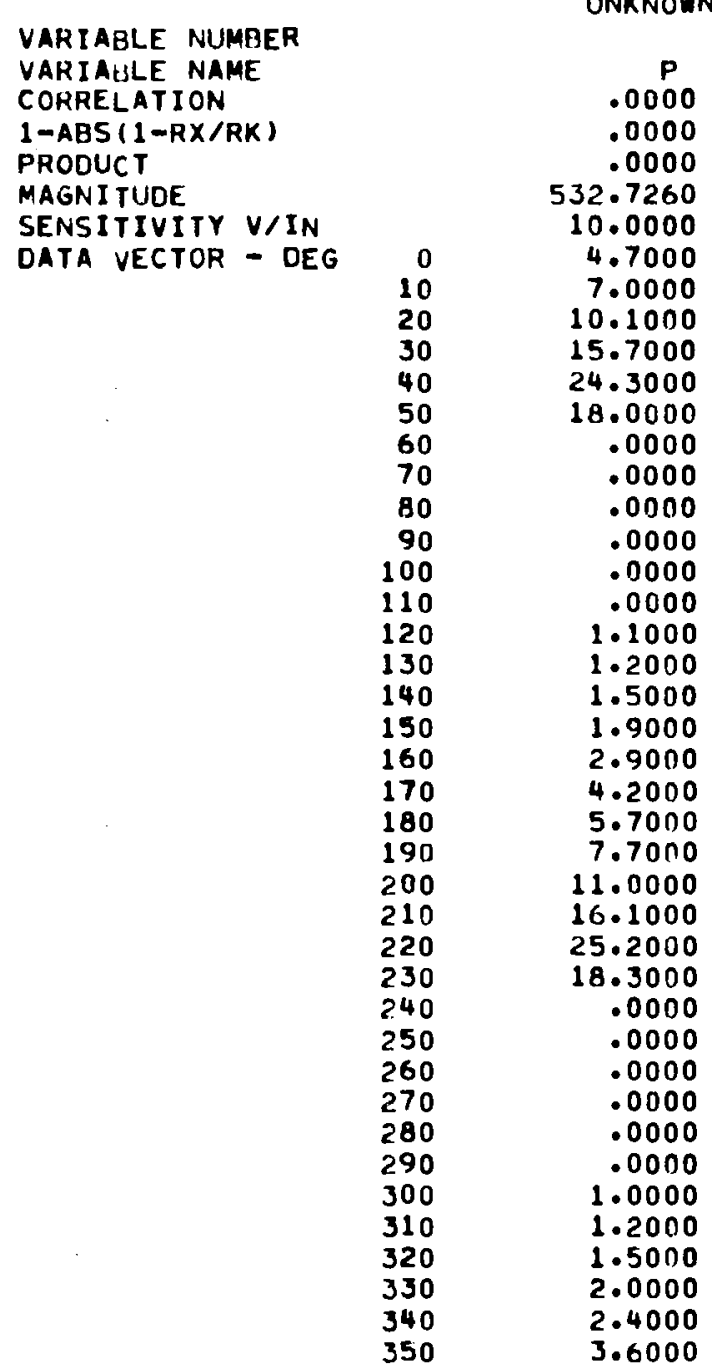

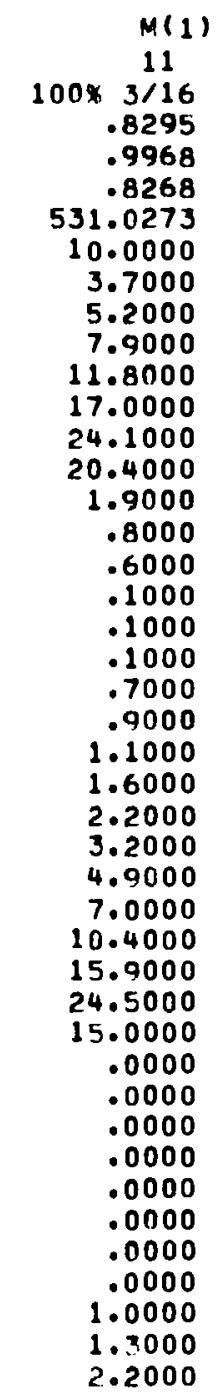

$M(2)$
15
$100 \% 118$
.8822
.1733
.1529
291.6316
10.0000
2.4000
3.4000
4.9000
6.9000
9.8000
13.2000
.0000
.0000
.0000
.0000
.0000
.0000
.0000
.0000
.0000
.0000
.0000
1.1000
1.9000
3.0000
4.5000
6.4000
9.9000
13.8000
11.0000
1.6000
.6000
.4000
.2000
.2000
.2000
.1000
.1000
.1000
1.0000
1.6000

$M(3)$
4
$20 \times 003 / 16$
.0395
-68.4662
-2.7061
7.5600
.2000
.0000
.0000
.0000
.0000
.0000
.0000
.0000
.0000
.0000
10.0000
9.4000
9.6000
10.0000
10.8000
11.03000
.0000
.0000
.0000
.0000
.0000
.0000
.0000
.0000
.0000
.0000
9.9000
10.9000
7.2000
8.7000
10.0000
11.2000
12.9000
7.0000
.0000
.0000
.0000

$M(4)$
20
$80 \times 10118$
.8094
-3.9989
-3.2365
88.8041
2.0000
.0000
2.0000
4.0000
6.9000
11.0000
18.4000
20.5000
.0000
.0000
.0000
.0000
.0000
.0000
.0000
.0000
.0000
.5000
1.0000
1.9000
4.0000
6.8000
10.1000
17.4000
23.5000
.0000
.0000
.0000
.0000
.0000
.0000
.0000
.0000
.0000
.0000
.0000
.0000

$M(5)$
19
$800 \quad 303116$
.8880
-3.8716
-3.4379
90.7290
2.0000
2.0000
3.2000
5.1000
1.9000
14.9000
27.0000
.0000
.0000
.0000
.0000
.0000
.0000
.0000
.0000
.0000
.0000
.5000
1.0000
1.8000
3.7000
5.3000
8.6000
14.1000
25.8000
1.0000
.0000
.0000
.0000
.0000
.0000
.0000
.0000
.0000
.3000
.7000
1.1000




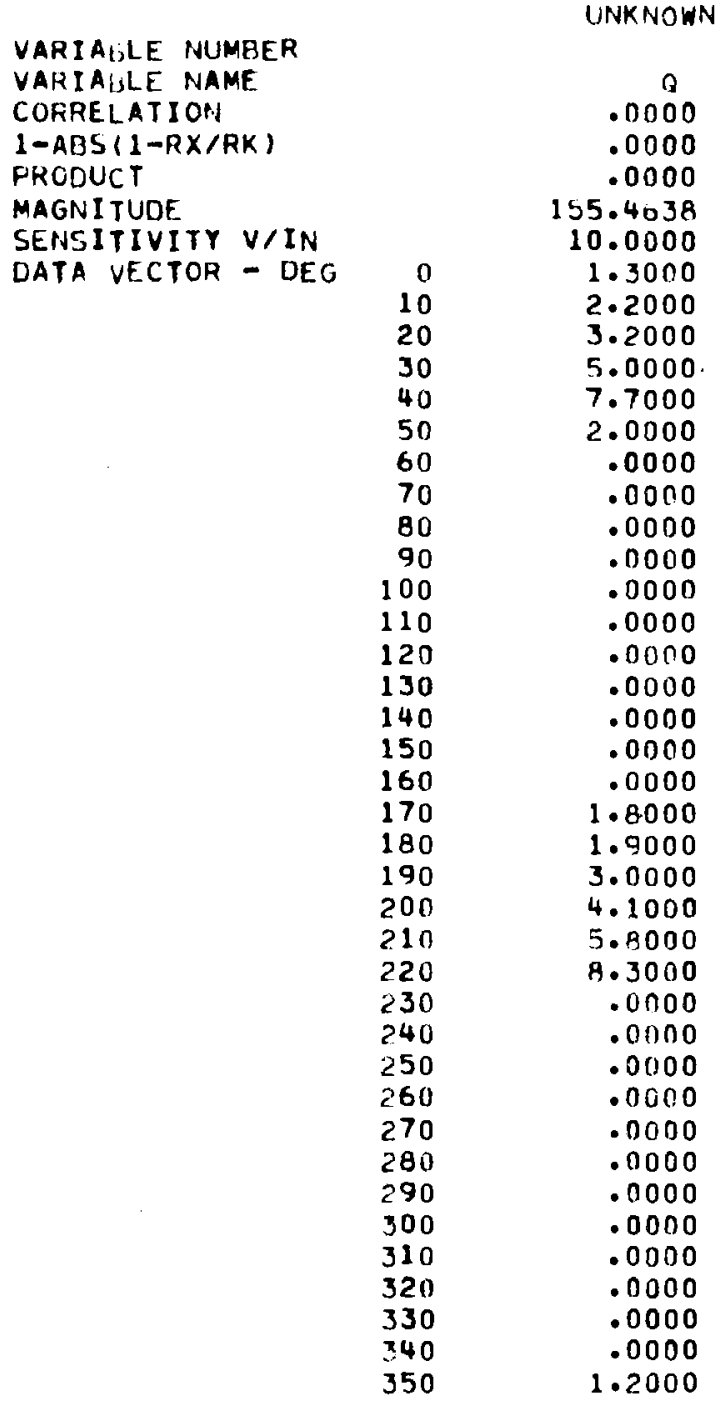

1111
15
$100 \times 118$
.6989
.5331
.3726
291.6316
10.0000
2.4000
3.4000
4.9000
6.9000
9.8000
13.2000
.0000
.0000
.0000
.0000
.0000
.0000
.0000
.0000
.0000
.0000
.0000
1.1000
1.9000
3.0000
4.5000
6.4000
9.9000
13.8000
11.0000
1.6000
.6000
.4000
.2000
.2000
.2000
.1000
.1000
.1000
1.0000
1.6000

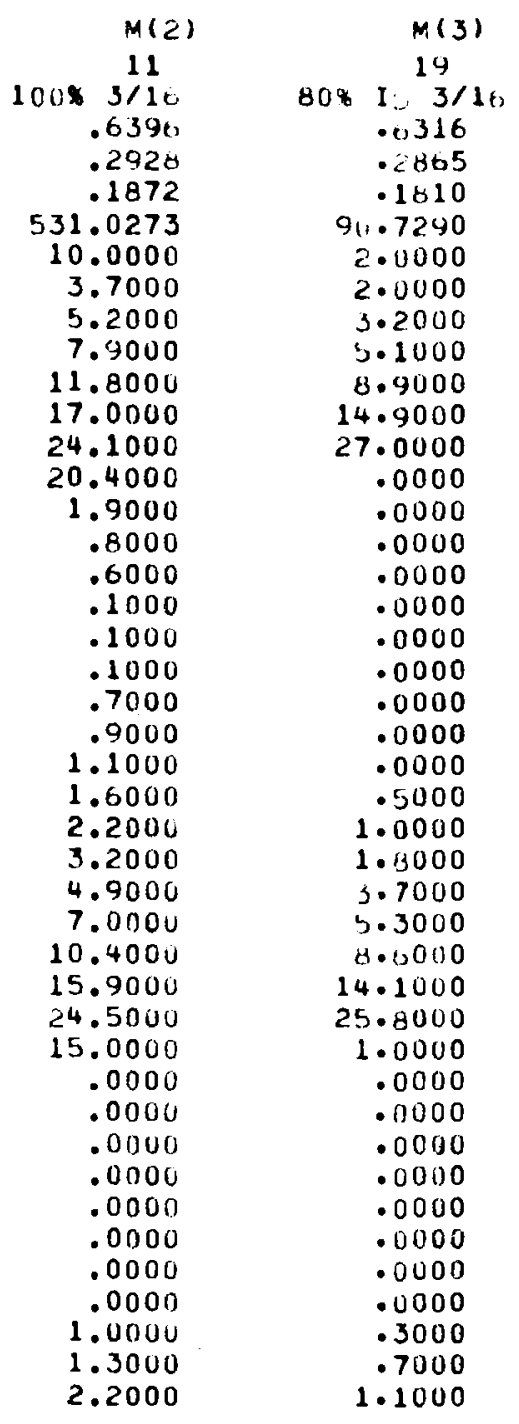

1141
14
1116
.8332
.2025
.1697
86.4884
2.0000
5.4000
7.2000
9.8000
12.9000
17.0000
19.9000
.5000
.5000
.0000
.7000
.8000
.9000
1.1000
1.2000
1.5000
2.0000
2.7000
3.3000
4.5000
6.1000
8.2000
11.6000
15.9000
16.0000
.0000
.0000
.0000
.0000
.5000
1.1000
1.3000
1.5000
1.9000
2.5000
3.2000
4.2000

1.90
1.90

$M 151$
220
1118
.0106
.2494
.1523
80.8041
2.0000
.0000
2.0000
4.0000
6.9010
11.0000
14.4000
20.5000
.0000
.0000
.0000
.0000
.0000
.0000
.0000
.0000
.0000
.5000
1.0000
1.9000
4.0000
0.8000
10.1000
17.4000
23.5000
.0000
.0000
.00000
.0000
.00000
.0000
.00100
.0000
.0000
.0000
.0000
.0000




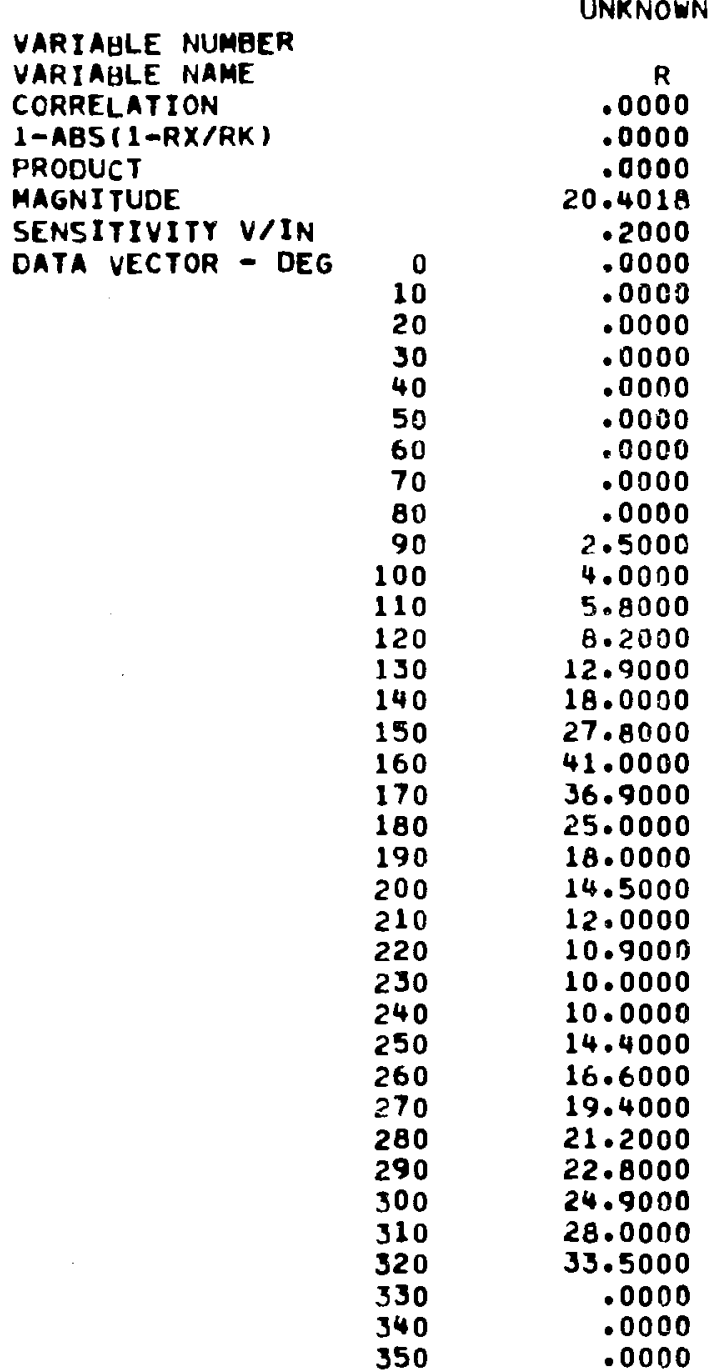

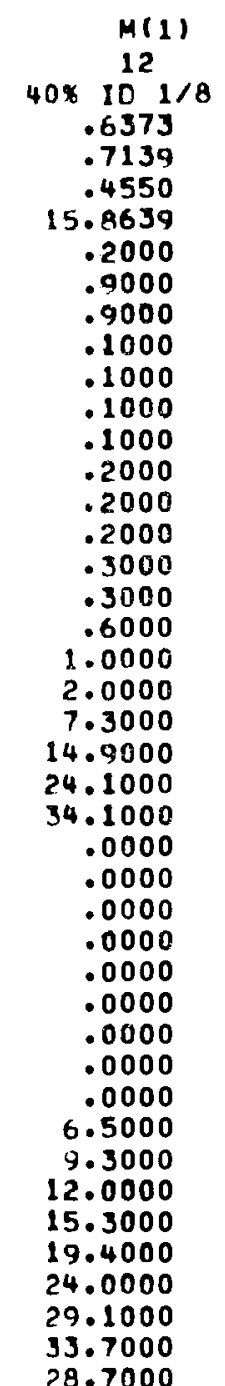

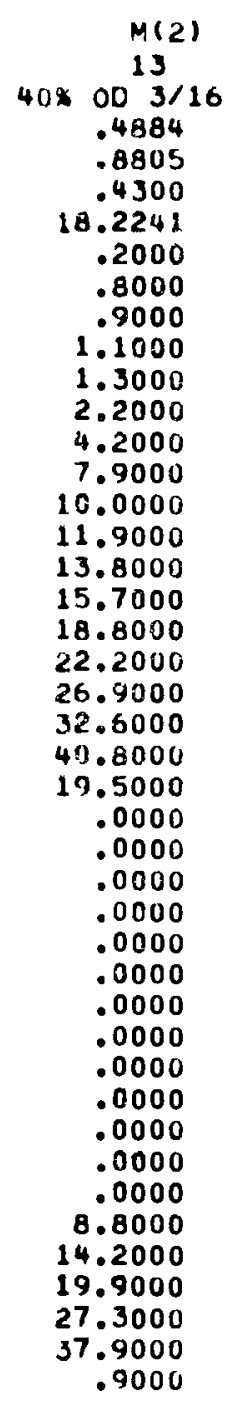

$M(3)$
8
10118
.3007
.9325
.2804
19.1114
.5000
.5000
.6000
.8000
.4000
1.2000
4.5000
11.0000
22.0000
.0000
.0000
1.5000
1.8000
1.4000
1.8000
1.8000
1.4000
2.0000
2.1000
2.4000
2.8000
3.2000
3.8000
5.5000
7.9000
12.3000
22.8000
.0000
1.2000
.7000
.5000
.4000
.4000
.3000
.3000
.3000
.4000

114
17
$50 \times 00.040 R$
.3991
.6311
.2519
14.9036
.5000
14.3000
1.0000
.2000
.0000
.0000
.0000
.0000
.0000
.0000
.0000
.0000
.0000
.0000
.0000
.0000
.0000
7.0000
10.8000
13.7000
1.0000
.0000
.0000
.0000
.0000
.0000
.0000
.0000
.0000
.0000
.0000
.0000
.0000
5.0000
7.2000
9.9000
12.4000

.000
.000
.000
.000
.000

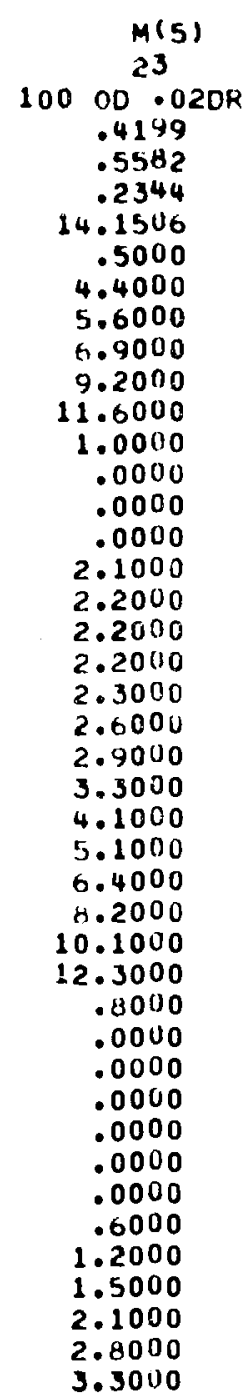


VARIATSLE NUIMBER

VARIATHLE VIAME

CORRELATION

$1-A B S(1-R X / R K)$

PRGDUCT

MAGNI TUDE

SENSITIVITY V/IN

OATA VECTOR - DEG
UNKNOWN

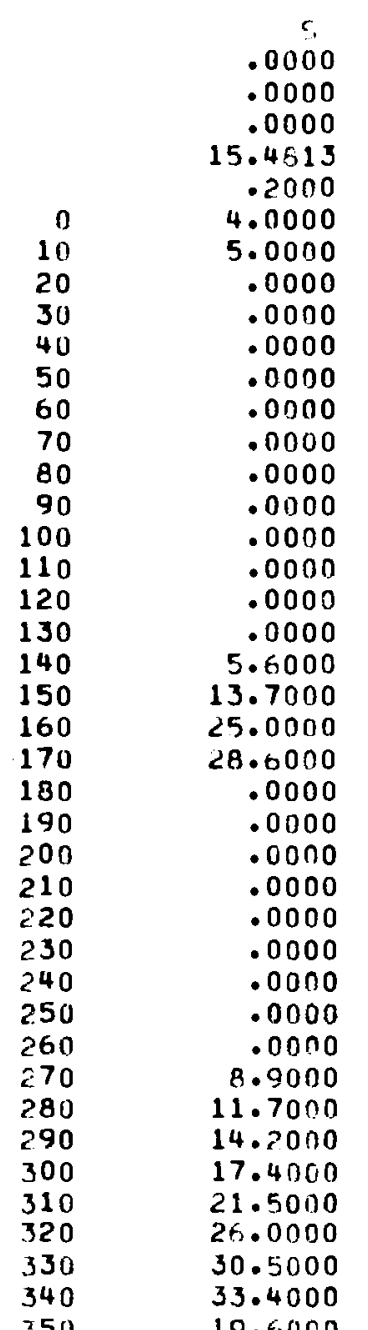

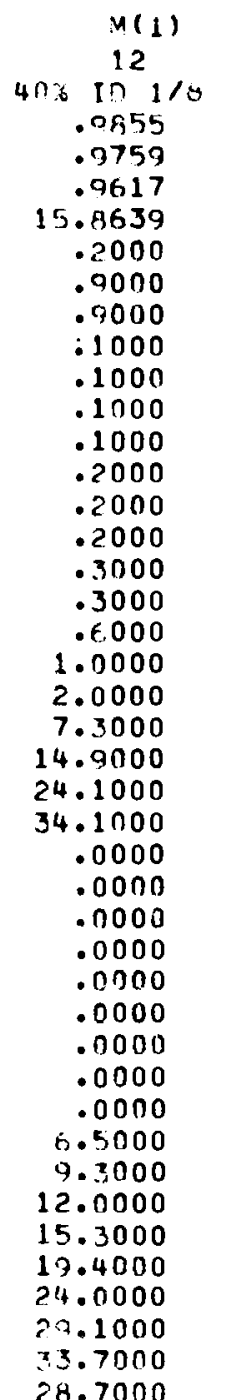

$M(2)$
17
509.0401
.6364
.9612
.6122
14.9036
.5000
14.3000
1.0000
.2000
.0000
.0000
.0000
.0000
.0000
.0000
.0000
.0000
.0000
.0000
.0000
.0000
.0000
7.0000
10.0000
13.7000
1.0000
.0000
.0000
.0000
.0000
.0000
.0000
.0000
.0000
.0000
.0000
.0000
.0000
5.0000
7.2000
9.9000
12.4000

$M(3)$
13
$40 \% .03 / 16$
.0137
.8495
.5213
16.2241
.2000
.8000
.9000
1.1000
1.3000
2.2000
4.2000
7.9000
10.0000
11.5000
13.5000
15.7000
18.0000
22.2000
26.9000
32.6000
40.8000
19.5000
.0000
.0000
.0000
.0000
.0000
.0000
.0000
.0000
.0000
.0000
.0000
.0000
.0000
6.8000
14.2000
19.9000
27.3060
37.9000
.9000

$M(4)$
16
$80.00 .020 K$
.6667
.5420
.3614
10.6184
.2000
17.6000
21.1000
1.0000
.4000
.0000
.0000
.0000
.0000
.0000
.0000
.9000
3.5000
5.9000
7.8000
9.3000
11.2000
13.0000
14.9000
17.1000
19.2000
1.8000
.0000
.0000
.0000
.0000
.0000
.0000
.0000
.0000
7.0000
7.2000
7.9000
8.0000
10.0000
12.0000
14.2000

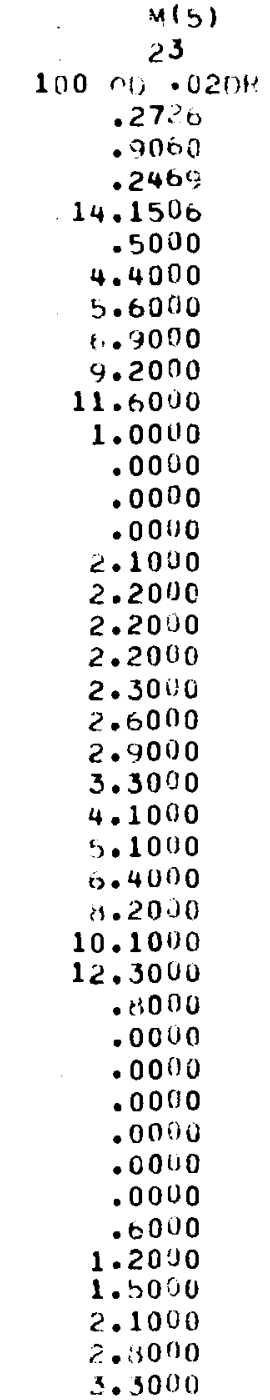




\begin{tabular}{|c|c|c|}
\hline $\begin{array}{l}\text { VARIABLE NUMBER } \\
\text { VARIAHLE NAME } \\
\text { CORRELATION } \\
\text { I-ABS (I-RX/RK) } \\
\text { PRODUCT } \\
\text { MAGNITUDE } \\
\text { SENSITIVITY V/IN } \\
\text { DATA VECTOR - DEG }\end{array}$ & $\begin{array}{r}0 \\
10 \\
20 \\
30 \\
40 \\
50 \\
60 \\
70 \\
80 \\
90 \\
100 \\
110 \\
120 \\
130 \\
140 \\
150 \\
160 \\
170 \\
180 \\
190 \\
200 \\
210 \\
220 \\
230 \\
240 \\
250 \\
260 \\
270 \\
280 \\
290 \\
300 \\
310 \\
320 \\
330 \\
340 \\
350\end{array}$ & $\begin{array}{r}T \\
.0000 \\
.0000 \\
.0000 \\
7.3150 \\
.2000 \\
2.0000 \\
1.9000 \\
1.9000 \\
1.9000 \\
2.0000 \\
2.0000 \\
2.2000 \\
2.7000 \\
4.3000 \\
.0000 \\
.0000 \\
5.0000 \\
7.2000 \\
9.1000 \\
11.1000 \\
13.7000 \\
16.0000 \\
10.0000 \\
.9000 \\
1.0000 \\
1.1000 \\
1.5000 \\
2.0000 \\
2.5000 \\
4.5000 \\
7.2000 \\
.0000 \\
.0000 \\
.0000 \\
.0000 \\
.0000 \\
.0000 \\
.0000 \\
9.2000 \\
14.3000 \\
9.0000\end{array}$ \\
\hline
\end{tabular}

$M(1)$
10
4081116
.9142
.9907
.9057
7.3838
.2000
2.5000
2.3000
2.1000
2.0000
.0000
.0000
.0000
.0000
.0000
.0000
.0000
.0000
5.0000
7.5000
9.9000
12.4000
15.1000
15.8000
.0000
.0000
.0000
.0000
.0000
.0000
.0000
.0000
.0000
.0000
.0000
.0000
.0000
.0000
.0000
9.4000
14.9000
14.0000

$M(2)$
18
$50800.020 R$
.5674
.9728
.5520
7.5194
.2000
8.8000
9.0000
9.0000
8.4000
7.4000
6.8000
6.3000
6.0000
5.9000
5.9000
6.0000
6.1000
6.6000
7.0000
8.0000
9.1000
.0000
.0000
.0000
.0000
.0000
.0000
.0000
.0000
.0000
.0000
.0000
2.5000
7.8000
8.1000
8.3000
6.1000
8.1000
8.1000
8.5000
8.6000

$M 131$
16
$800.020 R$
.6445
.6889
.4440
10.6184
.2000
17.6000
21.1000
1.0000
.4000
.0000
.0000
.0000
.0000
.0000
.0000
.9000
3.5000
5.9000
7.8000
9.3000
11.2000
13.0000
14.9000
17.1000
19.2000
1.8000
.6000
.0000
.0000
.0000
.0000
.0000
.0000
.0000
7.0000
7.2000
7.9000
8.8000
10.0000
12.0000
14.2000

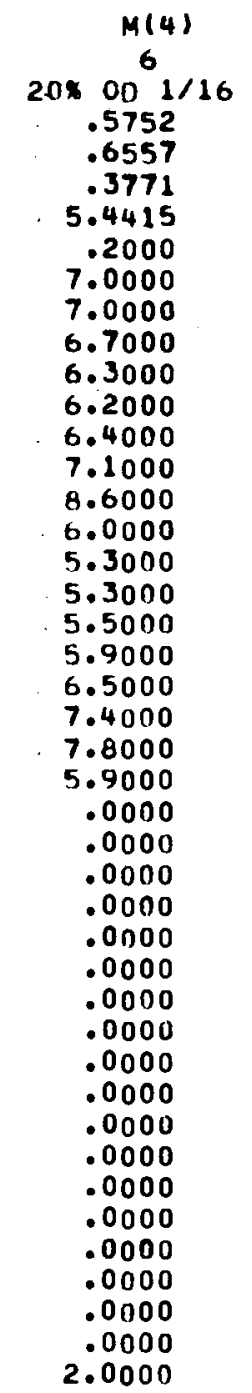

$M(5)$
12
$40 x .101 / 8$
.7081
.4611
.3265
15.8639
.2000
.9000
.9000
.1000
.1000
.1000
.1000
.2000
.2000
.2000
.3000
.3000
.6000
1.0000
2.0000
7.3000
14.9000
24.1000
34.1000
.0000
.0000
.0000
.0000
.0000
.0000
.0000
.0000
.0000
6.5000
9.3000
12.0000
15.3000
19.4000
24.0000
29.1000
33.7000
28.7000


APPENDIX III

Eddy Current Tubing Flaw Patterns

Intentional Flaws

1

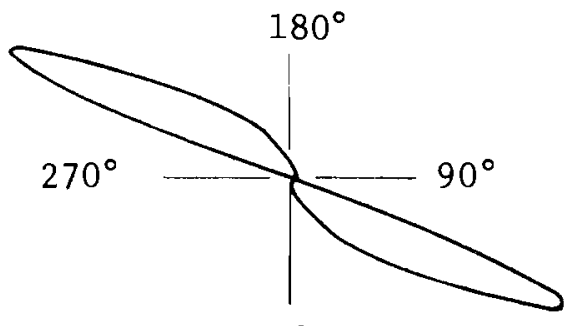

$40 \%$ ID Notch

$0.125^{\prime \prime}$ Long

2

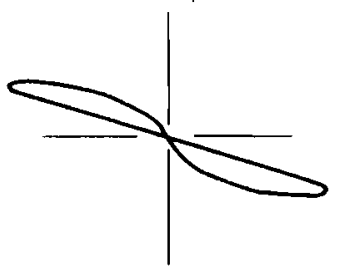

40\% ID Notch

$0.0625^{\prime \prime}$ Long

3

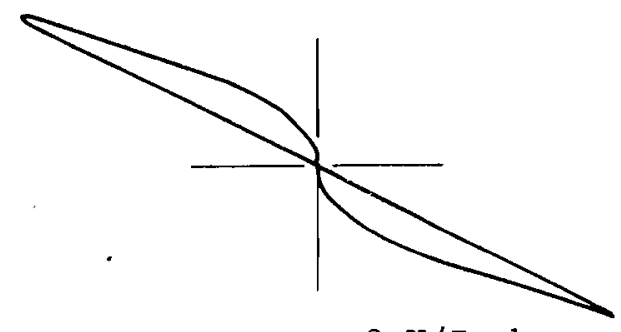

$40 \%$ ID Notch

$0.1875^{\prime \prime}$ Long

4

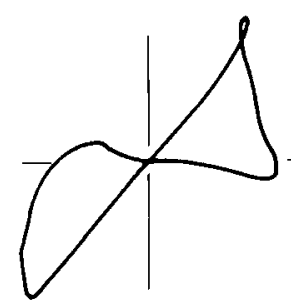

$20 \%$ OD Notch

$0.1875^{\prime \prime}$ Long
$2 \mathrm{~V} /$ Inch

$2 \mathrm{~V} /$ Inch

$0.4 \mathrm{~V} /$ Inch
5

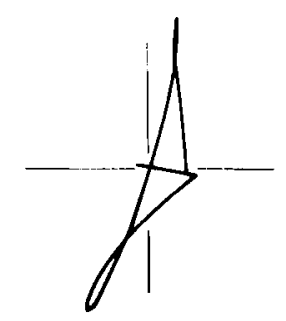

$20 \%$ oD Notch $0.125^{\prime \prime}$ Long

$0.4 \mathrm{~V} /$ Inch

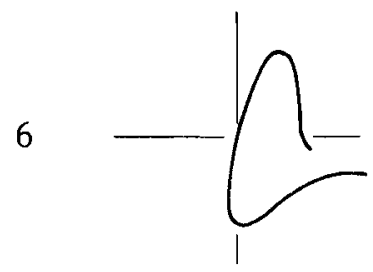

$20 \%$ OD Notch $0.0625^{\prime \prime}$ Long

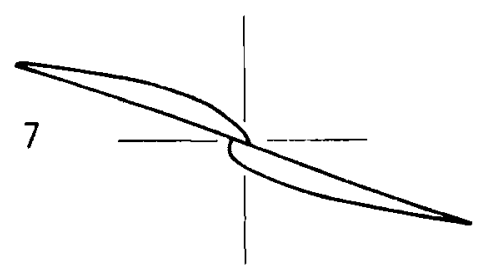

$20 \%$ ID Notch $0.1875^{\prime \prime}$ Long

$1.0 \mathrm{~V} /$ Inch

$0.4 \mathrm{~V} /$ Inch

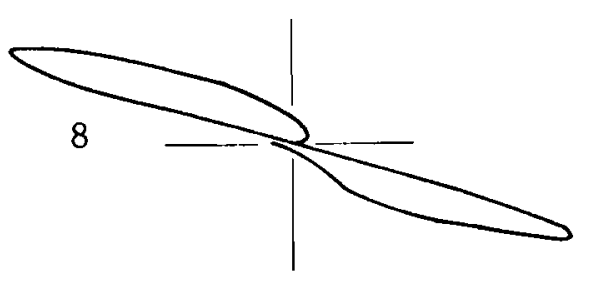

$20 \%$ ID Notch $1.0 \mathrm{~V} /$ Inch $0.125^{\prime \prime}$ Long 
APPENDIX III

Eddy Current Tubing Flaw Patterns

Intentional Flaws

9

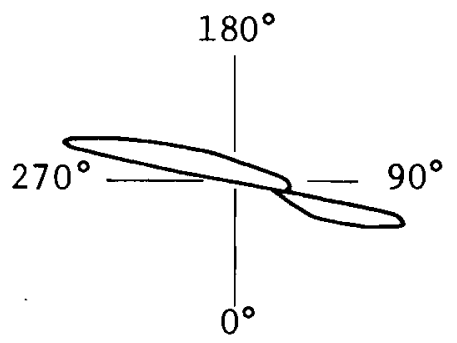

$20 \%$ ID Notch $0.0625^{\prime \prime}$ Long

10

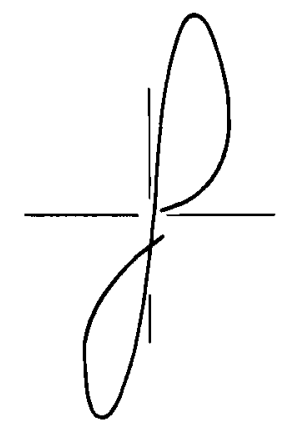

$40 \%$ OD Notch

$0.0625^{\prime \prime}$ Long

.11

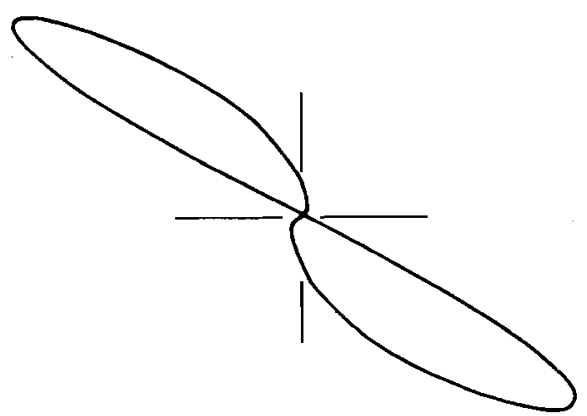

$100 \%$ Notch

$0.1875^{\prime \prime}$ Long

12

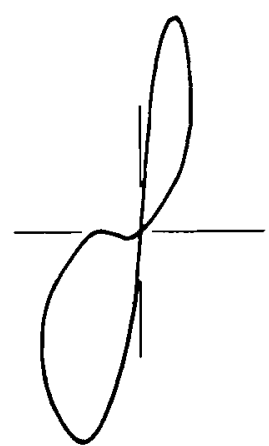

$40 \%$ OD Notch

0.125 " Long
$1.0 \mathrm{~V} /$ Inch

$0.4 \mathrm{~V} /$ Inch

$20 \mathrm{~V} /$ Inch

$0.8 \mathrm{~V} /$ Inch
13

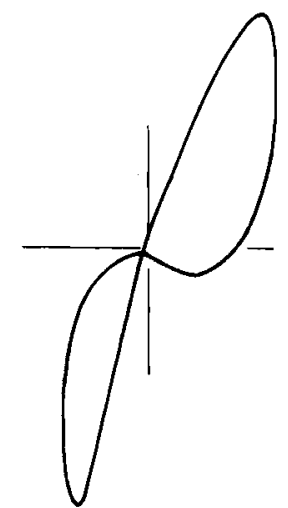

40\% OD Notch

$0.1875^{\prime \prime}$ Long

$0.8 \mathrm{~V} / \mathrm{cm}$

14

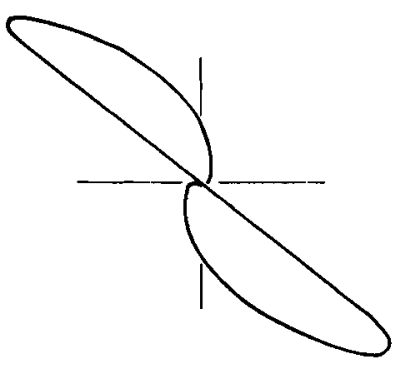

$100 \%$ Notch

$0.0625^{\prime \prime}$ Long

$4 \mathrm{~V} /$ Inch

15

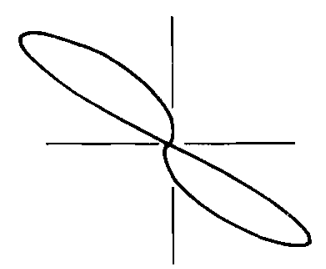

$100 \%$ Notch

0.125 " Long

$20 \mathrm{~V} /$ Inch

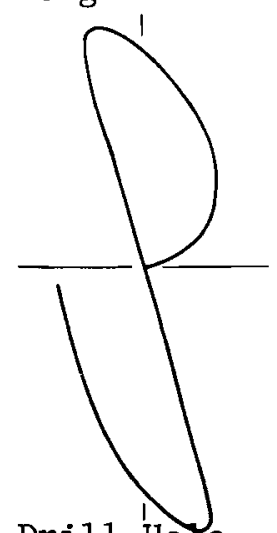

80\% OD Drill Hole

$0.020^{\prime \prime}$

$0.4 \mathrm{~V} /$ Inch 


\section{APPENDIX III}

Eddy Current Tubing Flaw Patterns Intentional Flaws

17

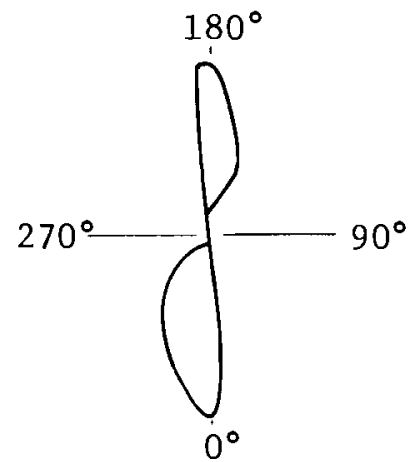

21

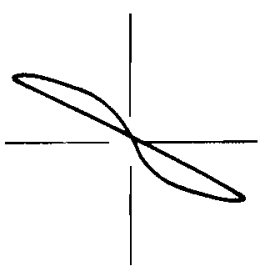

50\% OD Drill Hole

$1.0 \mathrm{~V} /$ Inch $0.040^{\prime \prime}$

18

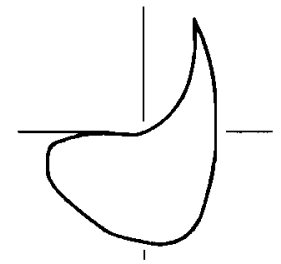

$0.4 \mathrm{~V} /$ Inch

50\% OD Drill Hole $0.020^{\prime \prime}$

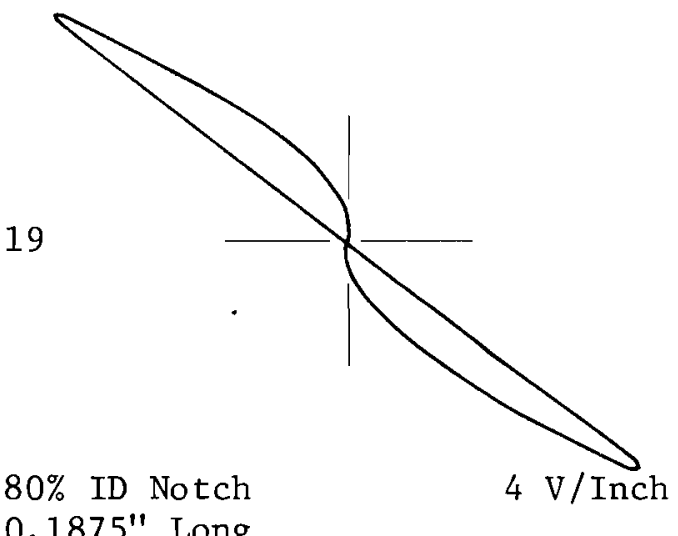

$0.1875^{\prime \prime}$ Long

20

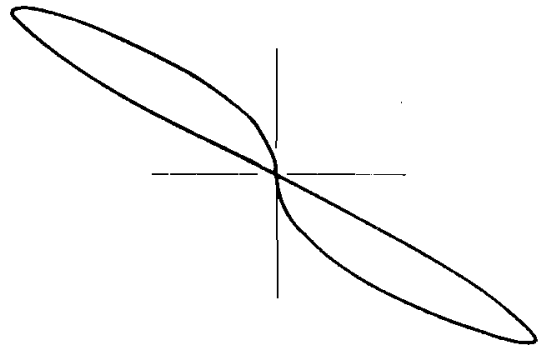

23

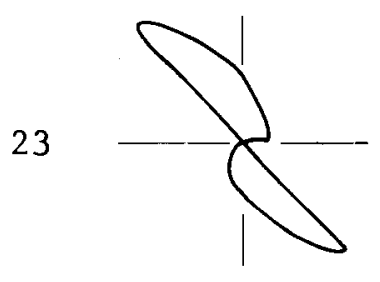

100\% OD Drill

Hole 0.040"

$2 \mathrm{~V} /$ Inch

$80 \%$ ID Notch

$4 \mathrm{~V} /$ Inch

22

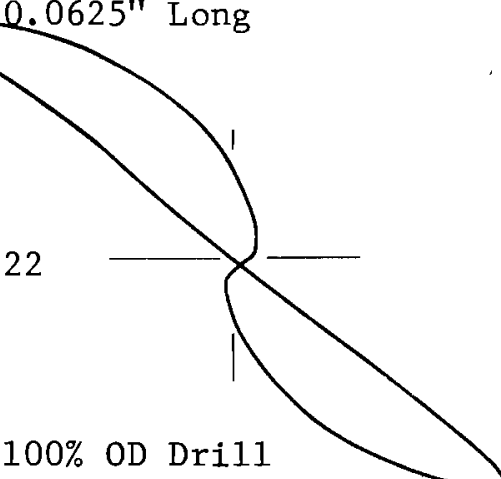

$100 \%$ OD Drill Hole $1.0 \mathrm{~V} /$ Inch $0.020^{\prime \prime}$ 


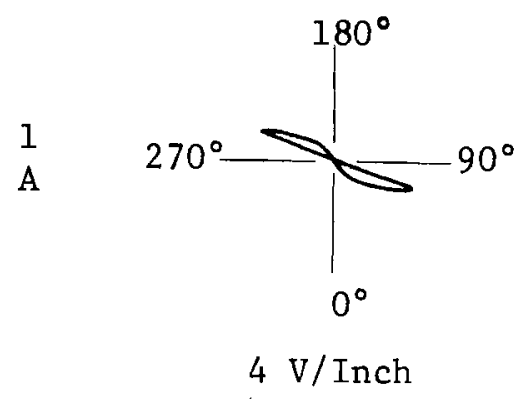

5
$E$

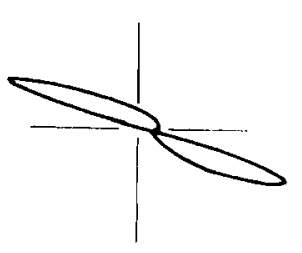

$2 \mathrm{~V} /$ Inch
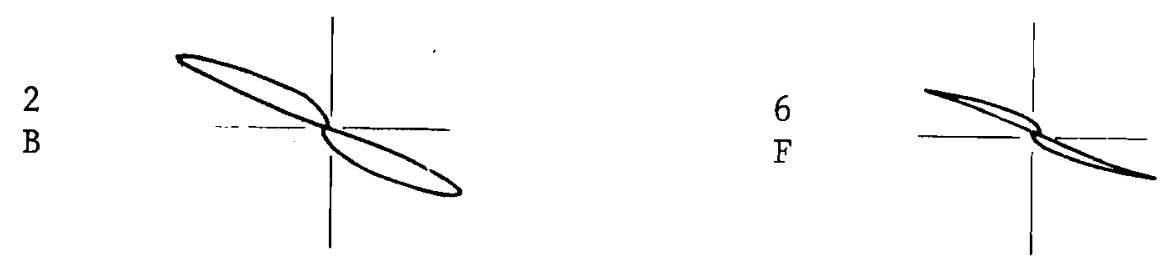

$4 \mathrm{~V} /$ Inch

$2 \mathrm{~V} /$ Inch

3

C

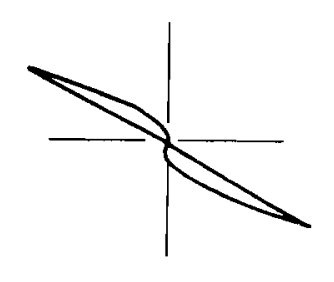

$4 \mathrm{~V} /$ Inch

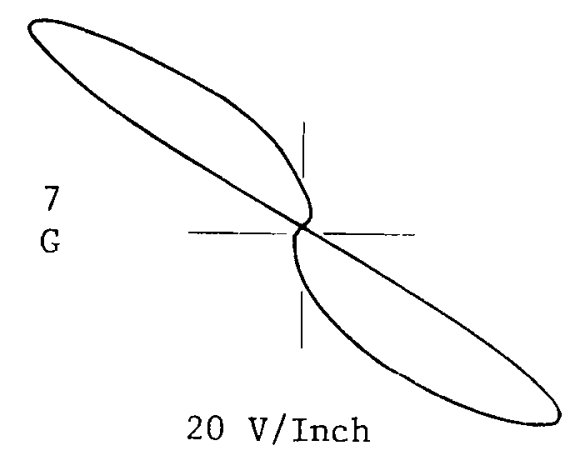

4

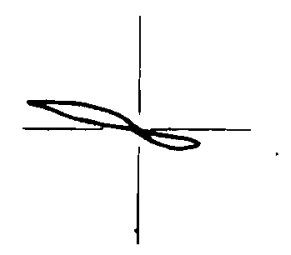

$2 \mathrm{~V} /$ Inch

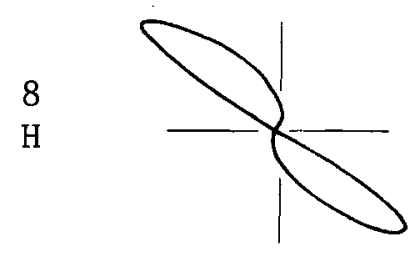

$20 \mathrm{~V} /$ Inch 


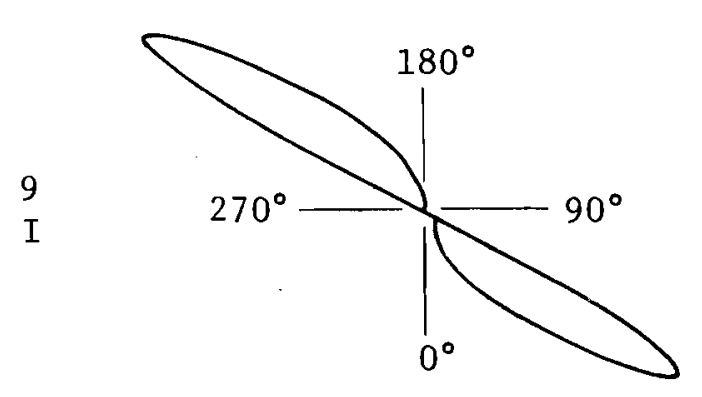

$4 \mathrm{~V} /$ Inch

10

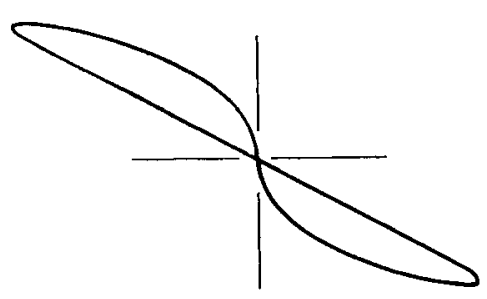

$2 \mathrm{~V} /$ Inch

11

$\mathrm{K}$

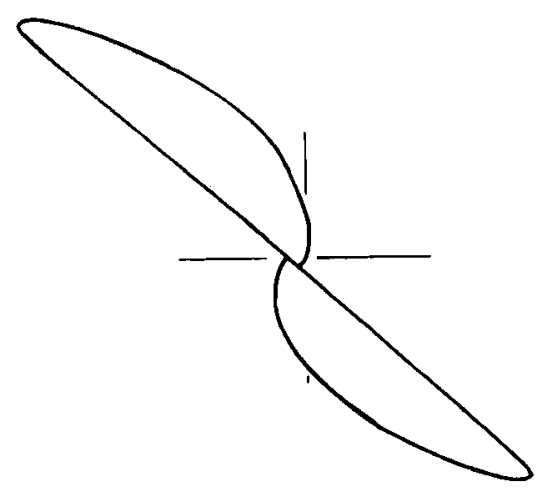

$2 \mathrm{~V} /$ Inch

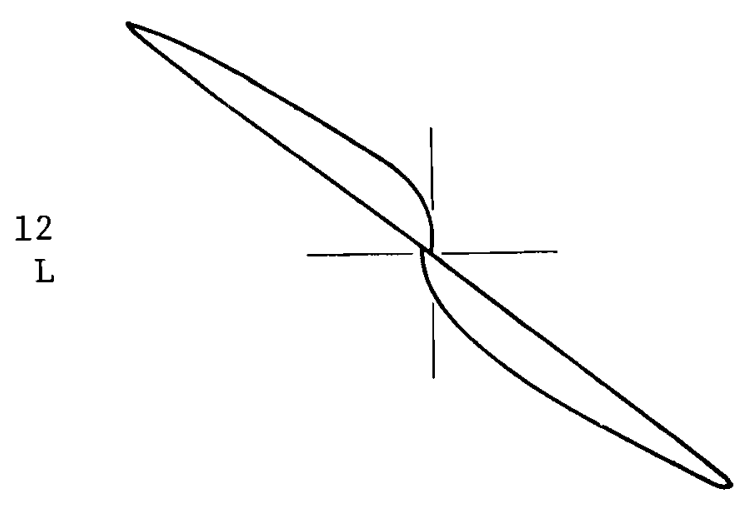

$4 \mathrm{~V} /$ Inch
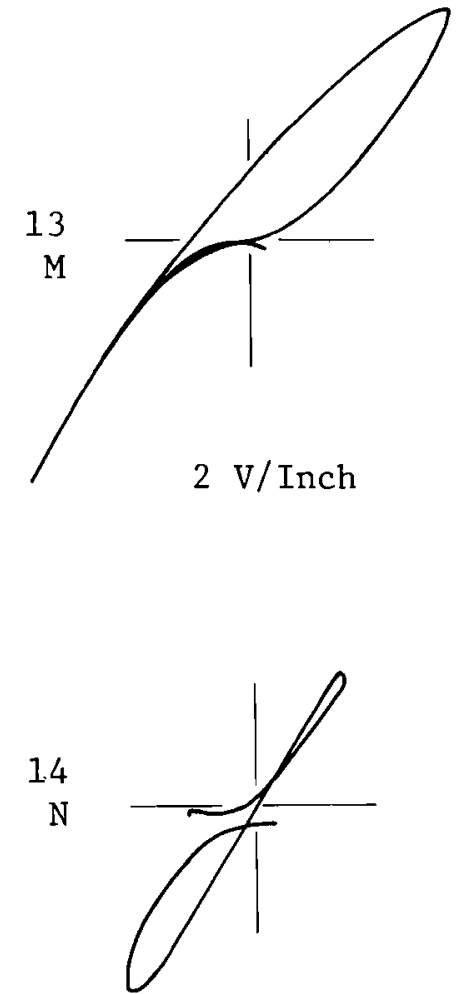

$2 \mathrm{~V} /$ Inch

15

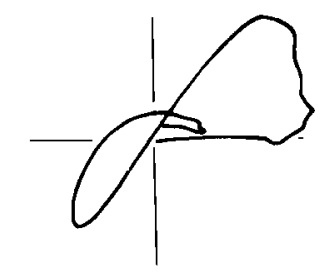

0

$2 \mathrm{~V} /$ Inch

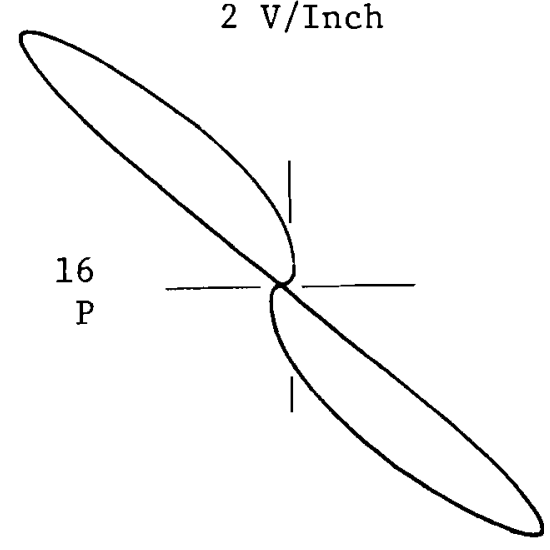

$20 \mathrm{~V} /$ Inch 

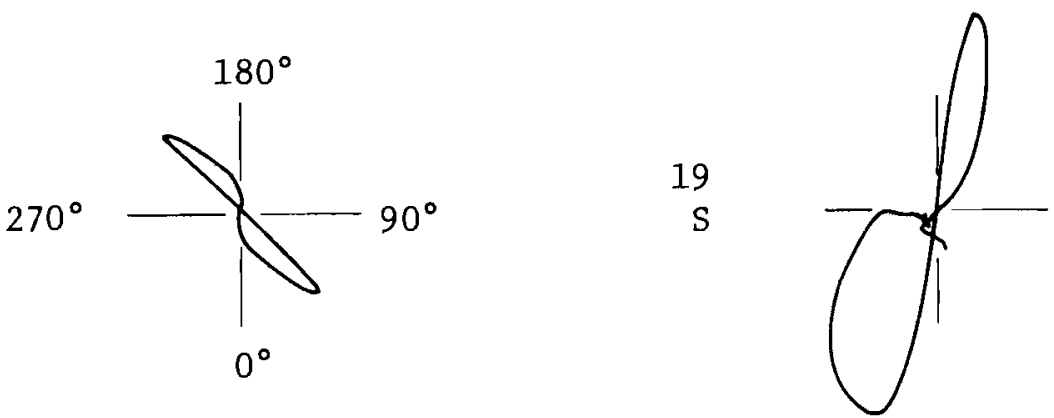

$$
20 \mathrm{~V} / \text { Inch }
$$
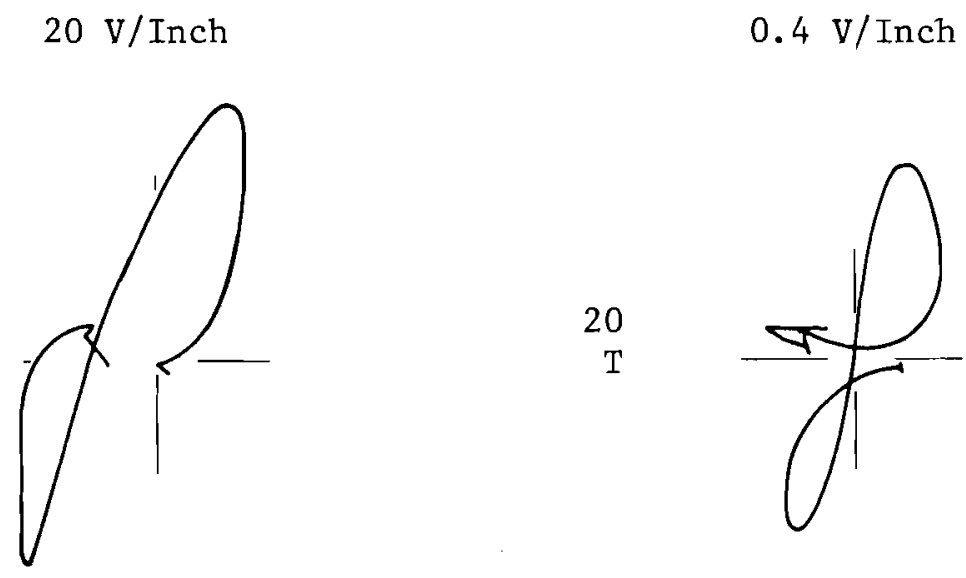

$$
0.4 \mathrm{~V} / \text { Inch }
$$

$0.4 \mathrm{~V} /$ Inch

APPENDIX IV

Eddy Current Tubing Flaw Patterns Random Flaws 
PROGRAM LOCATE WAS WRITTEN BY MRS. CAROL L. LEWIS APPL IFD MATHEMATICS BATTELLE-NORTHWEST

DIMENSION $R(4), 23), R \times(41), 1 D(23,2), M(5), N D(5,3)$

101 FORMAT (1HI)

FORMAT $(4 X, 2 A 6,29 X, F 5.2,10 F 3,1 / 2 x, 26 F 3.1)$

FORMAT (A2,43X,F5.2,10F3,1/2X,26F3.1)

100 FORMAT $(1 \mathrm{H}, 34 \mathrm{X}, 7$ HUNKNOWN, $11 \mathrm{X}, 5(2 \mathrm{HM}(, \mathrm{I}, 1 \mathrm{H}), 11 \mathrm{X})$

99 FJRMAT $(5 x, 15$ HVARIABLE NUMBER, $33 x, 5(I 2,13 x)$

98 FORMAT $(5 X, 3 A 6,8 X, 6(F 10.4,5 X))$

98 FORMAT (5X,3A6,8X,6(F10.4,5X))

97 FORMAT $15 x, 19 H D A T A$ VECTOR - DEG , I3,4X,6(F10.4,5x)

$24 X, 13,4 X, 6(F 10,4,5 X))$

95 FORMAT $15 X, 13$ HVARIABLE NAME, $20 X, A 2,7 X, 5(2 A 6,3 \times) 1$

CALL SETEOF (\$10JO)

DATA (IND(I,J), J=1,3),I=1,5)/6HCORREL, GHATION,6H ,6H1-ASS1,

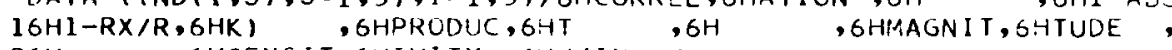

$26 \mathrm{H}$ , $6 \mathrm{HS}$

$S=0$

$\operatorname{READ}(5,1)(I D(I, K), K=1,2),(R(J, 1), J=5,41)$

DO $10 \mathrm{~J}=6,41$

$x=R(J, I)$

$10 \mathrm{~S}=\mathrm{C}+\mathrm{X} * \mathrm{X}$

$11 R(4,1)=\operatorname{SOR} T(5) * R(5, I)$

$22 \operatorname{READ}(5,2) \quad I X,(R X(j), J=5,41)$

DO $12 J=6.41$

$Y=R \times(J)$

$12 S=S+Y * Y$

$R \times(4)=S Q R T(S) * R \times(5)$

DO $14 \mathrm{I}=1,23$

$S=0$

DO $13 J=6,41$

$13 S=S+R \times(J) * R(J, I)$

$R(1, I)=S * R \times(5) * R(5, I) /(R \times(4) * R(4, I))$

$R(2, I)=1 .-A B S(1 .-R \times(4) / R(4, I)$

$14 R(3, I)=R(1, I) * R(2, I)$

DO $21 \quad 1=1$, 5 
30 WRITE $(6,98)(N D(J, I), I=1,3), R \times(J), R(J, M I), R(J, M 2), R(J, M 3)$, $1 R(J, M 4), R(J, M 5)$

WRITE $(6,97) K K, R \times(6), R\left(6, N_{1}\right), R(6, N, 2), R(6, M 3), R(6, M 4), R(6, M 5)$

DO $31 \mathrm{~J}=7,41$ $K K=K K+10$

31 WRITE $(6,96) K K, R \times(J), R(J, M 1), R(J, M 2), R(J, M 3), R(J, M 4), R(J, M 5)$ GO TO 22

OOO CONTINUE

NRITE $(6,101)$

STOP

END 


\section{DISTRIBUTION}

Number

of Copies

$\underline{\text { Off-Site }}$

AEC Chicago Patent Group

G. H. Lee

AEC Division of Reactor Development and Technology

J. M. Simmons

270

AEC Division of Technical Information Extension

Battelle Memorial Institute - Columbus

R. P. Heydorn

$\underline{\text { On-Site }}$

AEC Chicago Patent Group

R. K. Sharp

AEC RDT Site Representative

P. G. Holsted (2)

J. B. Kitchen

1

AEC Richland Operations Office

C. L. Robinson

Battelle-Northwest

W. G. Albert

C. A. Bennett

R. L. Brown

A. C. Callen

G. J. Dau

P. H. Hutton

J. L. Jaech

H. G. Johnson

W. D. Jo11y

L. T. Lamb

H. L. Libby

R. J. Lobsinger

W. R. McSpadden

M. H. Meuser

V. I. Neeley
R. N. Ord (10)

H. N. Pedersen (2)

J. A. Perry

H. G. Powers

R. L. Richardson (10)

R. L. Roske

C. B. Shaw

F. R. Shober

N. B. Smith

J. C. Spanner

D. D. Taylor

M. R. Wood

D. C. Worlton

Technical Files (5)

Technical Publications 
